Article

\title{
Dynamic Hilbert-Type Inequalities with Fenchel-Legendre Transform
}

\author{
Ahmed A. El-Deeb ${ }^{1, *}$, Samer D. Makharesh ${ }^{1}$ and Dumitru Baleanu ${ }^{2,3,4}$ (D)
}

1 Department of Mathematics, Faculty of Science, Al-Azhar University, Nasr City, Cairo 11884, Egypt; sameeermakarish@yahoo.com

2 Department of Mathematics, Cankaya University, Ankara 06530, Turkey; dumitru@cankaya.edu.tr or Baleanu@mail.cmuh.org.tw

3 Institute of Space Science, 07650 Magurele-Bucharest, Romania

4 Department of Medical Research, China Medical University Hospital, China Medical University, Taichung 40402, Taiwan

* Correspondence: ahmedeldeeb@azhar.edu.eg

Received: 12 March 2020; Accepted: 26 March 2020; Published: 7 April 2020

Abstract: Our work is based on the multiple inequalities illustrated in 2020 by Hamiaz and Abuelela. With the help of a Fenchel-Legendre transform, which is used in various problems involving symmetry, we generalize a number of those inequalities to a general time scale. Besides that, in order to get new results as special cases, we will extend our results to continuous and discrete calculus.

Keywords: Hilbert's inequality; Fubini theorem; Fenchel-Legendre transform; time scale

AMS Subject Classifications: 26D10; 26D15; 26E70; 34A40

\section{Introduction}

In 2020, Hamiaz and Abuelela [1] have studied the following discrete inequalities:

Theorem 1. Suppose $q, p \geqslant 1, \alpha \geqslant \beta \geqslant \frac{1}{2}$ and $\left(b_{m}\right)_{m} \geq 0\left(a_{n}\right)_{n} \geq 0$ are sequences of real numbers. Define $A_{n}=\sum_{s=1}^{n} a_{s}, B_{m}=\sum_{t=1}^{m} b_{t}$. Then

$$
\begin{array}{r}
\sum_{n=1}^{k} \sum_{m=1}^{r} \frac{A_{n}^{2 p} B_{m}^{2 q}}{h(n)+h^{*}(m)} \leqslant C_{1}^{*}(p, q)\left(\sum_{n=1}^{k}(k-n+1)\left(a_{n} A_{n}^{p-1}\right)^{2}\right) \\
\times\left(\sum_{m=1}^{r}(r-m+1)\left(b_{m} B_{m}^{q-1}\right)^{2}\right)
\end{array}
$$

and

$$
\begin{aligned}
\sum_{n=1}^{k} \sum_{m=1}^{r} \frac{A_{n}^{p} B_{m}^{q}}{\left(|h(n)|^{\frac{1}{2 \beta}}+\left|h^{*}(m)\right|^{\frac{1}{2 \beta}}\right)^{\alpha}} & \leqslant \sum_{n=1}^{k} \sum_{m=1}^{r} \frac{A_{n}^{p} B_{m}^{q}}{\sqrt{h(n)+h^{*}(m)}} \\
& \leqslant C_{2}^{*}(p, q, k, r)\left(\sum_{n=1}^{k}(k-n+1)\left(a_{n} A_{n}^{p-1}\right)^{2}\right)^{\frac{1}{2}} \\
& \times\left(\sum_{m=1}^{r}(r-m+1)\left(b_{m} B_{m}^{q-1}\right)^{2}\right)^{\frac{1}{2}}
\end{aligned}
$$


unless $\left(a_{n}\right)$ or $\left(b_{m}\right)$ is null, where

$$
C_{1}^{*}(p, q)=(p q)^{2} \text { and } C_{2}^{*}(p, q, r, k)=p q \sqrt{k r} .
$$

Hilger [2] suggested time scales theory to unify discrete and continuous analysis. More Hilbert-type inequalities and other types can be seen in [1,3-36], see also [37-53]. For more details on time scales calculus see [54].

We will need the following important relations between calculus on time scales $\mathbb{T}$ and either continuous calculus on $\mathbb{R}$ or discrete calculus on $\mathbb{Z}$. Note that:

(i) If $\mathbb{T}=\mathbb{R}$, then

$$
\sigma(t)=t, \quad \mu(t)=0, \quad f^{\Delta}(t)=f^{\prime}(t), \quad \int_{a}^{b} f(t) \Delta t=\int_{a}^{b} f(t) d t
$$

(ii) If $\mathbb{T}=\mathbb{Z}$, then

$$
\sigma(t)=t+1, \quad \mu(t)=1, \quad f^{\Delta}(t)=f(t+1)-f(t), \quad \int_{a}^{b} f(t) \Delta t=\sum_{t=a}^{b-1} f(t) .
$$

Next is Hölder's and Jensen's inequality:

Lemma 1 ([19]). Let $a, b \in \mathbb{T}$ and $f, g \in C_{r d}\left([a, b]_{\mathbb{T}},[0, \infty)\right)$. If $p, q>1$ with $\frac{1}{p}+\frac{1}{q}=1$, then

$$
\int_{a}^{b} f(t) g(t) \Delta t \leq\left[\int_{a}^{b} f^{p}(t) \Delta t\right]^{\frac{1}{p}}\left[\int_{a}^{b} g^{q}(t) \Delta t\right]^{\frac{1}{q}}
$$

Lemma 2 ( [19]). Let $a, b \in \mathbb{T}$ and $\breve{c}, \breve{d} \in \mathbb{R}$. Assume that $g \in C_{r d}\left([a, b]_{\mathbb{T}},[\breve{c}, \breve{d}]\right)$ and $r \in C_{r d}\left([a, b]_{\mathbb{T}}, \mathbb{R}\right)$ are nonnegative with $\int_{a}^{b} r(t) \Delta t>0$. If $\breve{\Phi} \in C_{r d}((\breve{c}, \breve{d}), \mathbb{R})$ be a convex function, then

$$
\breve{\Phi}\left(\frac{\int_{a}^{b} g(t) r(t) \Delta t}{\int_{a}^{b} r(t) \Delta t}\right) \leqslant \frac{\int_{a}^{b} r(t) \breve{\Phi}(g(t)) \Delta t}{\int_{a}^{b} r(t) \Delta t} .
$$

Now, we present the Fenchel-Legendre transform and refer, for example, to [11-13], for more details.

Definition 1. Assuming $h: \mathbb{R}^{n} \longrightarrow \mathbb{R} \cup\{+\infty\}$ is a function: $h \neq+\infty$ i.e., $\operatorname{Dom}(h)=\left\{x \in \mathbb{R}^{n}, \mid h(x)<\right.$ $\infty\} \neq \varnothing$. Then the Fenchel-Legendre transform is defined as:

$$
h^{*}: \mathbb{R}^{n} \longrightarrow \mathbb{R} \cup\{+\infty\}, y \longrightarrow h^{*}(y)=\sup \{<y, x>-h(x), x \in \operatorname{Dom}(h)\}
$$

where $\left\langle., .>\right.$ is the scalar product on $\mathbb{R}^{n}$. The mapping $h \longrightarrow h^{*}$ is often be called the conjugate operation.

The domain of $h^{*}$ is the set of slopes of all the affine functions minorizing the function $h$ over $\mathbb{R}^{n}$. An equivalent formula for (3) is introduced as follows:

Corollary 1. Assuming $h: \mathbb{R}^{n} \longrightarrow \mathbb{R}$ is differentiable, strictly convex and 1-coercive function. Then

$$
h^{*}(y)=<y_{,}(\nabla h)^{-1}(y)>-h\left((\nabla h)^{-1}(y)\right),
$$

$\forall y \in \operatorname{Dom}\left(h^{*}\right)$, where $<. . .>$ denotes the scalar product on $\mathbb{R}^{n}$. 
Lemma 3 ( [13]). Let $h$ be a function and $h^{*}$ its Fenchel-Legendre transform. Then

$$
<x, y>\leqslant h(x)+h^{*}(y)
$$

for all $x \in \operatorname{Dom}(h)$, and $y \in \operatorname{Dom}\left(h^{*}\right)$.

In addition, we will use the following definition and lemma as we will see in the proof of our results:

Definition 2. The function $\breve{\Phi}$ is said to be a submultiplicative on $[0, \infty)$ if

$$
\breve{\Phi}(x y) \leqslant \breve{\Phi}(x) \breve{\Phi}(y), \text { for all } x, y \geqslant 0 \text {. }
$$

Lemma 4 ([20]). Assuming $\mathbb{T}$ is a time scale with $x, a \in \mathbb{T}$ such that $x \geqslant a$. If $f \geqslant 0$ and $\tilde{\alpha} \geqslant 1$, then

$$
\left(\int_{a}^{\sigma(x)} f(\check{\tau}) \Delta \check{\tau}\right)^{\tilde{\alpha}} \leqslant \tilde{\alpha} \int_{a}^{\sigma(x)} f(\eta)\left(\int_{a}^{\sigma(\eta)} f(\check{\tau}) \Delta \check{\tau}\right)^{\tilde{\alpha}-1} \Delta \eta
$$

Next, we write Fubini's theorem on time scales.

Lemma 5 (Fubini's Theorem, see [55]). Assume that $\left(X, \Sigma_{1}, \mu_{\Delta}\right)$ and $\left(Y, \Sigma_{2}, v_{\Delta}\right)$ are two finite-dimensional time scales measure spaces. Moreover, suppose that $f: X \times Y \rightarrow \mathbb{R}$ is a delta integrable function and define the functions

$$
\hat{\pi}_{1}(y)=\int_{X} f(x, y) d \mu_{\Delta}(x), \quad y \in Y,
$$

and

$$
\hat{\pi}_{2}(x)=\int_{X} f(x, y) d v_{\Delta}(y), \quad x \in X .
$$

Then $\hat{\pi}_{1}$ is delta integrable on $Y$ and $\hat{\pi}_{2}$ is delta integrable on $X$ and

$$
\int_{X} d \mu_{\Delta}(x) \int_{Y} f(x, y) d v_{\Delta}(y)=\int_{Y} d v_{\Delta}(y) \int_{X} f(x, y) d \mu_{\Delta}(x) .
$$

In this manuscript, by using Fubini's theorem and the Fenchel-Legendre transform, which is used in various problems involving symmetry, we extend the discrete results proved in [1] on time scales. We start from the inequalities treated in the Theorem 1 . Our results can be applied to give more general forms of some previously proved inequalities through substituting $h$ and $h^{*}$ by suitable functions as we will see in the following two sections.

The following section contains our main results.

\section{Main Results}

We start by establishing the following useful inequality:

Lemma 6. Assume $x$ and $y \in \mathbb{R}$ such that $x+y \geqslant 1$, then for $\gamma>0$, and $\alpha \geqslant \beta \geqslant \frac{1}{2}$, we get

$$
(x+y)^{\frac{1}{\gamma}} \leqslant\left(|x|^{\frac{1}{2 \beta}}+|y|^{\frac{1}{2 \beta}}\right)^{\frac{2 \alpha}{\gamma}} .
$$

Proof. For $x+y \geqslant 1$ and $\frac{\alpha}{\beta} \geqslant 1$, we have

$$
(x+y)^{\frac{1}{2}} \leqslant\left[(x+y)^{\frac{1}{2}}\right]^{\frac{\alpha}{\beta}}=\left[(x+y)^{\frac{1}{2 \beta}}\right]^{\alpha} \leqslant\left[(|x|+|y|)^{\frac{1}{2 \beta}}\right]^{\alpha} .
$$


From $(|x|+|y|)^{\frac{1}{n}} \leqslant|x|^{\frac{1}{n}}+|y|^{\frac{1}{n}}$, for all $n \geqslant 1$. Thus, from (9), and since $2 \beta \geqslant 1$, we obtain:

$$
(x+y)^{\frac{1}{2}} \leqslant\left[(|x|+|y|)^{\frac{1}{2 \beta}}\right]^{\alpha} \leqslant\left[|x|^{\frac{1}{2 \beta}}+|y|^{\frac{1}{2 \beta}}\right]^{\alpha} .
$$

Now, since $\gamma>0$, by taking the power $1 / \gamma$ for both sides of (10), we get:

$$
(x+y)^{\frac{1}{\gamma}} \leqslant\left(|x|^{\frac{1}{2 \beta}}+|y|^{\frac{1}{2 \beta}}\right)^{\frac{2 \alpha}{\gamma}} .
$$

This proves our claim.

In the next theorems, we will let $p>1, q>1$ and $\frac{1}{q}+\frac{1}{p}=1$.

Theorem 2. Let $\mathbb{T}$ be a time scale with $L \geqslant 1, K \geqslant 1$ and $s, t, t_{0}, x, y \in \mathbb{T}$. Assume $a(\check{\tau}) \geq 0$ and $b(\check{\tau}) \geq 0$ are right-dense continuous functions on the time scales intervals $\left[t_{0}, x\right]_{\mathbb{T}}$ and $\left[t_{0}, y\right]_{\mathbb{T}}$ respectively and define

$$
A(s):=\int_{t_{0}}^{s} a(\check{\tau}) \Delta \check{\tau} \text {, and } B(t):=\int_{t_{0}}^{t} b(\check{\tau}) \Delta \check{\tau},
$$

then for $\sigma(s) \in\left[t_{0}, x\right]_{\mathbb{T}}$ and $\sigma(t) \in\left[t_{0}, y\right]_{\mathbb{T}}$, we have that

$$
\begin{aligned}
& \int_{t_{0}}^{x} \int_{t_{0}}^{y} \frac{A^{q K}(\sigma(s)) B^{q L}(\sigma(t))}{\left(\left|h\left(\sigma(s)-t_{0}\right)\right|^{\frac{1}{2 \beta}}+\left|h^{*}\left(\sigma(t)-t_{0}\right)\right|^{\frac{1}{2 \beta}}\right)^{\frac{2 q \alpha}{p}}} \Delta s \Delta t \\
& \leqslant C_{1}(L, K, q)\left(\int_{t_{0}}^{x}(\sigma(x)-\sigma(s))\left(a(s) A^{K-1}(\sigma(s))^{q} \Delta s\right)\right. \\
& \times\left(\int_{t_{0}}^{y}(\sigma(y)-\sigma(t))\left(b(t) B^{L-1}(\sigma(t))^{q} \Delta t\right)\right.
\end{aligned}
$$

and

$$
\begin{aligned}
& \int_{t_{0}}^{x} \int_{t_{0}}^{y} \frac{A^{K}(\sigma(s)) B^{L}(\sigma(t))}{\left(\left|h\left(\sigma(s)-t_{0}\right)\right|^{\frac{1}{2 \beta}}+\left|h^{*}\left(\sigma(t)-t_{0}\right)\right|^{\frac{1}{2 \beta}}\right)^{\frac{2 \alpha}{p}}} \Delta s \Delta t \\
& \leqslant C_{2}(L, K, p)\left(\int_{t_{0}}^{x}(\sigma(x)-\sigma(s))\left(A^{K-1}(\sigma(s)) a(s)\right)^{q} \Delta s\right)^{\frac{1}{q}} \\
& \times\left(\int_{t_{0}}^{y}(\sigma(y)-\sigma(t))\left(B^{L-1}(\sigma(t)) b(t)\right)^{q} \Delta t\right)^{\frac{1}{q}}
\end{aligned}
$$

where $C_{1}(L, K, q)=(K L)^{q}$ and $C_{2}(L, K, p)=K L\left(x-t_{0}\right)^{\frac{1}{p}}\left(y-t_{0}\right)^{\frac{1}{p}}$.

Proof. By using the inequality (7), we obtain

$$
\begin{aligned}
& A^{K}(\sigma(s)) \leqslant K \int_{t_{0}}^{\sigma(s)} a(\eta) A^{K-1}(\sigma(\eta) \Delta \eta, \\
& B^{L}(\sigma(t)) \leqslant L \int_{t_{0}}^{\sigma(t)} b(\eta) B^{L-1}(\sigma(\eta)) \Delta \eta .
\end{aligned}
$$


We use Lemma 1. Then from (13), we get

$$
A^{K}(\sigma(s)) \leqslant K\left(\sigma(s)-t_{0}\right)^{\frac{1}{p}}\left(\int_{t_{0}}^{\sigma(s)}\left(a(\eta) A^{K-1}(\sigma(\eta))^{q} \Delta \eta\right)^{\frac{1}{q}} .\right.
$$

We use Lemma 1. Then from (14), we also have

$$
B^{L}(\sigma(t)) \leqslant L\left(\sigma(t)-t_{0}\right)^{\frac{1}{p}}\left(\int_{t_{0}}^{\sigma(t)}\left(b(\eta) B^{L-1}(\sigma(\eta))^{q} \Delta \eta\right)^{\frac{1}{q}}\right.
$$

From (15) and (16), we get

$$
\begin{aligned}
A^{K}(\sigma(s)) B^{L}( & \sigma(t)) \leqslant K L\left(\sigma(s)-t_{0}\right)^{\frac{1}{p}}\left(\sigma(t)-t_{0}\right)^{\frac{1}{p}} \\
& \times\left(\int_{t_{0}}^{\sigma(s)}\left(a(\eta) A^{K-1}(\sigma(\eta))^{q} \Delta \eta\right)^{\frac{1}{q}}\right. \\
& \times\left(\int_{t_{0}}^{\sigma(t)}\left(b(\eta) B^{L-1}(\sigma(\eta))^{q} \Delta \eta\right)^{\frac{1}{q}} .\right.
\end{aligned}
$$

From inequality (17), we have

$$
\begin{aligned}
A^{q K}(\sigma(s)) B^{q L}(\sigma(t)) & \leqslant(K L)^{q}\left(\sigma(s)-t_{0}\right)^{\frac{q}{p}}\left(\sigma(t)-t_{0}\right)^{\frac{q}{p}} \\
& \times\left(\int_{t_{0}}^{\sigma(s)}\left(a(\eta) A^{K-1}(\sigma(\eta))^{q} \Delta \eta\right)\right. \\
& \times\left(\int_{t_{0}}^{\sigma(t)}\left(b(\eta) B^{L-1}(\sigma(\eta))^{q} \Delta \eta\right) .\right.
\end{aligned}
$$

Using Lemma 3 in (17) and (18) gives

$$
\begin{aligned}
& A^{K}(\sigma(s)) B^{L}(\sigma(t)) \leqslant K L\left(h\left(\sigma(s)-t_{0}\right)+h^{*}\left(\sigma(t)-t_{0}\right)\right)^{\frac{1}{p}} \\
& \times\left(\int_{t_{0}}^{\sigma(s)}\left(a(\eta) A^{K-1}(\sigma(\eta))^{q} \Delta \eta\right)^{\frac{1}{q}}\right. \\
& \times\left(\int_{t_{0}}^{\sigma(t)}\left(b(\eta) B^{L-1}(\sigma(\eta))^{q} \Delta \eta\right)^{\frac{1}{q}},\right. \\
& A^{q K}(\sigma(s)) B^{q L}(\sigma(t)) \leqslant(K L)^{q}\left(h\left(\sigma(s)-t_{0}\right)+h^{*}\left(\sigma(t)-t_{0}\right)\right)^{\frac{q}{p}} \\
& \times\left(\int_{t_{0}}^{\sigma(s)}\left(a(\eta) A^{K-1}(\sigma(\eta))^{q} \Delta \eta\right)\right. \\
& \times\left(\int_{t_{0}}^{\sigma(t)}\left(b(\eta) B^{L-1}(\sigma(\eta))^{q} \Delta \eta\right) .\right.
\end{aligned}
$$


Using Lemma 6 in (19) and (20) gives

$$
\begin{array}{r}
A^{K}(\sigma(s)) B^{L}(\sigma(t)) \leqslant K L\left(\left|h\left(\sigma(s)-t_{0}\right)\right|^{\frac{1}{2 \beta}}+\left|h^{*}\left(\sigma(t)-t_{0}\right)\right|^{\frac{1}{2 \beta}}\right)^{\frac{2 \alpha}{p}} \\
\times\left(\int_{t_{0}}^{\sigma(s)}\left(a(\eta) A^{K-1}(\sigma(\eta))^{q} \Delta \eta\right)^{\frac{1}{q}}\right. \\
\times\left(\int_{t_{0}}^{\sigma(t)}\left(b(\eta) B^{L-1}(\sigma(\eta))^{q} \Delta \eta\right)^{\frac{1}{q}}\right. \\
A^{q K}(\sigma(s)) B^{q L}(\sigma(t)) \leqslant(K L)^{q}\left(\left|h\left(\sigma(s)-t_{0}\right)\right|^{\frac{1}{2 \beta}}+\left|h^{*}\left(\sigma(t)-t_{0}\right)\right|^{\frac{1}{2 \beta}}\right)^{\frac{2 q \alpha}{p}} \\
\times\left(\int_{t_{0}}^{\sigma(s)}\left(a(\eta) A^{K-1}(\sigma(\eta))^{q} \Delta \eta\right)\right. \\
\times\left(\int_{t_{0}}^{\sigma(t)}\left(b(\eta) B^{L-1}(\sigma(\eta))^{q} \Delta \eta\right) .\right.
\end{array}
$$

Dividing both sides of (21) and (22) by $\left(\left|h\left(\sigma(s)-t_{0}\right)\right|^{\frac{1}{2 \beta}}+\left|h^{*}\left(\sigma(t)-t_{0}\right)\right|^{\frac{1}{2 \beta}}\right)^{\frac{2 \alpha}{p}}$ and $\left(\left|h\left(\sigma(s)-t_{0}\right)\right|^{\frac{1}{2 \beta}}+\right.$ $\left.\left|h^{*}\left(\sigma(t)-t_{0}\right)\right|^{\frac{1}{2 \beta}}\right)^{\frac{2 q \alpha}{p}}$ respectively, we get that

$$
\begin{aligned}
& \frac{A^{K}(\sigma(s)) B^{L}(\sigma(t))}{\left(\left|h\left(\sigma(s)-t_{0}\right)\right|^{\frac{1}{2 \beta}}+\left|h^{*}\left(\sigma(t)-t_{0}\right)\right|^{\frac{1}{2 \beta}}\right)^{\frac{2 \alpha}{p}} \leqslant} K L\left(\int_{t_{0}}^{\sigma(s)}\left(a(\eta) A^{K-1}(\sigma(\eta))^{q} \Delta \eta\right)^{\frac{1}{q}}\right. \\
& \times\left(\int_{t_{0}}^{\sigma(t)}\left(b(\eta) B^{L-1}(\sigma(\eta))^{q} \Delta \eta\right)^{\frac{1}{q}}\right. \\
& \frac{A^{q K}(\sigma(s)) B^{q L}(\sigma(t))}{\left(\left|h\left(\sigma(s)-t_{0}\right)\right|^{\frac{1}{2 \beta}}+\left|h^{*}\left(\sigma(t)-t_{0}\right)\right|^{\frac{1}{2 \beta}}\right)^{\frac{2 q \alpha}{p}} \leqslant(K L)^{q}\left(\int_{t_{0}}^{\sigma(s)}\left(a(\eta) A^{K-1}(\sigma(\eta))^{q} \Delta \eta\right)\right.} \\
& \times\left(\int_{t_{0}}^{\sigma(t)}\left(b(\eta) B^{L-1}(\sigma(\eta))^{q} \Delta \eta\right) .\right.
\end{aligned}
$$

From (23) by using Lemma 1 we obtain

$$
\begin{aligned}
& \int_{t_{0}}^{x} \int_{t_{0}}^{y} \frac{A^{K}(\sigma(s)) B^{L}(\sigma(t))}{\left(\left|h\left(\sigma(s)-t_{0}\right)\right|^{\frac{1}{2 \beta}}+\left|h^{*}\left(\sigma(t)-t_{0}\right)\right|^{\frac{1}{2 \beta}}\right)^{\frac{2 \alpha}{p}}} \Delta s \Delta t \\
& \leqslant K L\left(x-t_{0}\right)^{\frac{1}{p}}\left(y-t_{0}\right)^{\frac{1}{p}} \int_{t_{0}}^{x}\left(\int_{t_{0}}^{\sigma(s)}\left(a(\eta) A^{K-1}(\sigma(\eta))^{q} \Delta \eta\right) \Delta s\right)^{\frac{1}{q}} \\
& \times \int_{t_{0}}^{y}\left(\int_{t_{0}}^{\sigma(t)}\left(b(\eta) B^{L-1}(\sigma(\eta))^{q} \Delta \eta\right) \Delta t\right)^{\frac{1}{q}} .
\end{aligned}
$$


From (24), we get

$$
\begin{array}{r}
\int_{t_{0}}^{x} \int_{t_{0}}^{y} \frac{A^{q K}(\sigma(s)) B^{q L}(\sigma(t))}{\left(\left|h\left(\sigma(s)-t_{0}\right)\right|^{\frac{1}{2 \beta}}+\left|h^{*}\left(\sigma(t)-t_{0}\right)\right|^{\frac{1}{2 \beta}}\right)^{\frac{2 q \alpha}{p}} \Delta s \Delta t} \\
\leqslant(K L)^{q} \int_{t_{0}}^{x}\left(\int_{t_{0}}^{\sigma(s)}\left(a(\eta) A^{K-1}(\sigma(\eta))^{q} \Delta \eta\right) \Delta s\right) \\
\quad \times \int_{t_{0}}^{y}\left(\int_{t_{0}}^{\sigma(t)}\left(b(\eta) B^{L-1}(\sigma(\eta))^{q} \Delta \eta\right) \Delta t\right) .
\end{array}
$$

Applying Fubini's Theorem on (25) and (26) gives

$$
\begin{aligned}
& \int_{t_{0}}^{x} \int_{t_{0}}^{y} \frac{A^{K}(\sigma(s)) B^{L}(\sigma(t))}{\left(\left|h\left(\sigma(s)-t_{0}\right)\right|^{\frac{1}{2 \beta}}+\left|h^{*}\left(\sigma(t)-t_{0}\right)\right|^{\frac{1}{2 \beta}}\right)^{\frac{2 \alpha}{p}}} \Delta s \Delta t \\
& \leqslant K L\left(x-t_{0}\right)^{\frac{1}{p}}\left(y-t_{0}\right)^{\frac{1}{p}}\left(\int_{t_{0}}^{x}(x-\sigma(s))\left(a(s) A^{K-1}(\sigma(s))^{q} \Delta s\right)^{\frac{1}{q}}\right. \\
& \times\left(\int_{t_{0}}^{y}(y-\sigma(t))\left(b(t) B^{L-1}(\sigma(t))^{q} \Delta t\right)^{\frac{1}{q}}\right. \\
& \int_{t_{0}}^{x} \int_{t_{0}}^{y} \frac{A^{q K}(\sigma(s)) B^{q L}(\sigma(t))}{\left(\left|h\left(\sigma(s)-t_{0}\right)\right|^{\frac{1}{2 \beta}}+\left|h^{*}\left(\sigma(t)-t_{0}\right)\right|^{\frac{1}{2 \beta}}\right)^{\frac{2 q \alpha}{p}} \Delta s \Delta t} \\
& \leqslant(K L)^{q}\left(\int_{t_{0}}^{x}(x-\sigma(s))\left(a(s) A^{K-1}(\sigma(s))^{q}\right) \Delta t\right) \\
& \times\left(\int_{t_{0}}^{y}(y-\sigma(t))\left(b(t) B^{L-1}(\sigma(t))^{q} \Delta t\right) .\right.
\end{aligned}
$$

Using the facts $\sigma(x) \geqslant x, \sigma(y) \geqslant y$ yields

$$
\begin{aligned}
& \int_{t_{0}}^{x} \int_{t_{0}}^{y} \frac{A^{K}(\sigma(s)) B^{L}(\sigma(t))}{\left(\left|h\left(\sigma(s)-t_{0}\right)\right|^{\frac{1}{2 \beta}}+\left|h^{*}\left(\sigma(t)-t_{0}\right)\right|^{\frac{1}{2 \beta}}\right)^{\frac{2 \alpha}{p}}} \Delta s \Delta t \\
& \leqslant C_{2}(L, K, p)\left(\int_{t_{0}}^{x}(\sigma(x)-\sigma(s))\left(a(s) A^{K-1}(\sigma(s))^{q} \Delta s\right)^{\frac{1}{q}}\right. \\
& \times\left(\int_{t_{0}}^{y}(\sigma(y)-\sigma(t))\left(b(t) B^{L-1}(\sigma(t))^{q} \Delta t\right)^{\frac{1}{q}},\right. \\
& \int_{t_{0}}^{x} \int_{t_{0}}^{y} \frac{A^{q K}(\sigma(s)) B^{q L}(\sigma(t))}{\left(\left|h\left(\sigma(s)-t_{0}\right)\right|^{\frac{1}{2 \beta}}+\left|h^{*}\left(\sigma(t)-t_{0}\right)\right|^{\frac{1}{2 \beta}}\right)^{\frac{2 q \alpha}{p}}} \Delta s \Delta t \\
& \leqslant C_{1}(L, K, q)\left(\int_{t_{0}}^{x}(\sigma(x)-\sigma(s))\left(a(s) A^{K-1}(\sigma(s))^{2} \Delta s\right)\right. \\
& \times\left(\int_{t_{0}}^{y}(\sigma(y)-\sigma(t))\left(b(t) B^{L-1}(\sigma(t))^{q} \Delta t\right) .\right.
\end{aligned}
$$


This completes the proof.

Theorem 3. Let $a(\check{\tau}), b(\eta), A(s)$ and $B(t)$ be defined as in Theorem 2, thus

$$
\begin{aligned}
& \int_{t_{0}}^{x} \int_{t_{0}}^{y} \frac{A^{q}(\sigma(s)) B^{q}(\sigma(t))}{\left(\left|h\left(\sigma(s)-t_{0}\right)\right|^{\frac{1}{2 \beta}}+\left|h^{*}\left(\sigma(t)-t_{0}\right)\right|^{\frac{1}{2 \beta}}\right)^{\frac{2 q \alpha}{p}}} \Delta s \Delta t \\
& \leqslant\left(\int_{t_{0}}^{x}(\sigma(x)-\sigma(s)) a^{q}(s) \Delta s\right)\left(\int_{t_{0}}^{y}(\sigma(y)-\sigma(t)) b^{q}(t) \Delta t\right)
\end{aligned}
$$

and

$$
\begin{aligned}
& \int_{t_{0}}^{x} \int_{t_{0}}^{y} \frac{A(\sigma(s)) B(\sigma(t))}{\left(\left|h\left(\sigma(s)-t_{0}\right)\right|^{\frac{1}{2 \beta}}+\left|h^{*}\left(\sigma(t)-t_{0}\right)\right|^{\frac{1}{2 \beta}}\right)^{\frac{2 \alpha}{p}}} \Delta s \Delta t \\
& \leqslant\left(x-t_{0}\right)^{\frac{1}{p}}\left(y-t_{0}\right)^{\frac{1}{p}}\left(\int_{t_{0}}^{x}(\sigma(x)-\sigma(s)) a^{q}(s) \Delta s\right)^{\frac{1}{q}}\left(\int_{t_{0}}^{y}(\sigma(y)-\sigma(t)) b^{q}(t) \Delta t\right)^{\frac{1}{q}} .
\end{aligned}
$$

Proof. Put $K=L=1$ in (11) and (12). This completes the proof.

In Theorem 2, if we choose $\mathbb{T}=\mathbb{R}$, then we have relation (1) and the next results:

Corollary 2. If $a(s) \geq 0, b(t) \geq 0$. Define $A(s):=\int_{0}^{s} a(\eta) d \eta$ and $B(t):=\int_{0}^{t} b(\eta) d \eta$, then

$$
\begin{aligned}
& \int_{0}^{x} \int_{0}^{y} \frac{A^{q K}(s) B^{q L}(t)}{\left(|h(s)|^{\frac{1}{2 \beta}}+\left|h^{*}(t)\right|^{\frac{1}{2 \beta}}\right)^{\frac{2 q \alpha}{p}}} d s d t \\
& \leqslant C_{1}(L, K, q)\left(\int_{0}^{x}(x-s)\left(a(s) A^{K-1}(s)\right)^{q} d s\right) \\
& \times\left(\int_{0}^{y}(y-t)\left(b(t) B^{L-1}(t)\right)^{q} d t\right) .
\end{aligned}
$$

and

$$
\begin{aligned}
& \int_{0}^{x} \int_{0}^{y} \frac{A^{K}(s) B^{L}(t)}{\left(|h(s)|^{\frac{1}{2 \beta}}+\left|h^{*}(t)\right|^{\frac{1}{2 \beta}}\right)^{\frac{2 \alpha}{p}}} d s d t \\
& \leqslant C_{3}(L, K, p)\left(\int_{0}^{x}(x-s)\left(A^{K-1}(s) a(s)\right)^{q} d s\right)^{\frac{1}{q}} \\
& \times\left(\int_{0}^{y}(y-t)\left(B^{L-1}(t) b(t)\right)^{q} d t\right)^{\frac{1}{q}}
\end{aligned}
$$

where

$$
C_{3}(L, K, p)=K L(x y)^{\frac{1}{p}}
$$

In Theorem 2, if we chose $\mathbb{T}=\mathbb{Z}$, then we get (2), and the next result:

Corollary 3. If $a(n) \geq$ and $b(m) \geq 0$. Define

$$
A(n)=\sum_{s=0}^{n} a(s), \quad B(m)=\sum_{k=0}^{m} b(k) .
$$


Then

$$
\begin{array}{r}
\sum_{n=1}^{N} \sum_{m=1}^{M} \frac{A^{q L}(n) B^{q K}(m)}{\left(|h(n+1)|^{\frac{1}{2 \beta}}+\left|h^{*}(m+1)\right|^{\frac{1}{2 \beta}}\right)^{\frac{2 q \alpha}{p}}} \leqslant C_{1}(K, L, q)\left(\sum_{n=1}^{N}(N+1-(n+1))\left(a(n) A^{L-1}(n)\right)^{q}\right) \\
\\
\times\left(\sum_{m=1}^{M}(M+1-(m+1))\left(b(m) B^{L-1}(m)\right)^{q}\right)
\end{array}
$$

and

$$
\begin{aligned}
& \sum_{n=1}^{N} \sum_{m=1}^{M} \frac{A^{L}(n) B^{K}(m)}{\left(|h(n+1)|^{\frac{1}{2 \beta}}+\left|h^{*}(m+1)\right|^{\frac{1}{2 \beta}}\right)^{\frac{2 \alpha}{p}}} \leqslant C_{4}(K, L, p)\left(\sum_{n=1}^{N}(N+1-(n+1))\left(a(n) A^{L-1}(n)\right)^{q}\right)^{\frac{1}{q}} \\
& \times\left(\sum_{m=1}^{M}(M+1-(m+1))\left(b(m) B^{L-1}(m)\right)^{q}\right)^{\frac{1}{q}}
\end{aligned}
$$

where

$$
C_{4}(K, L, p)=K L(N M)^{\frac{1}{p}}
$$

Remark 1. Taking $p=q=2$ in Corollary 3 gives the result due to Hamiaz and Abuelela ([1], Theorem 3).

Corollary 4. With the hypotheses of Theorem 2 we have:

$$
\begin{aligned}
& \int_{t_{0}}^{x} \int_{t_{0}}^{y} \frac{A^{q K}(\sigma(s)) B^{q L}(\sigma(t))}{\left(\left|h\left(\sigma(s)-t_{0}\right)\right|^{\frac{1}{2 \beta}}+\left|h^{*}\left(\sigma(t)-t_{0}\right)\right|^{\frac{1}{2 \beta}}\right)^{\frac{2 q \alpha}{p}} \Delta s \Delta t} \\
& \leqslant C_{1}(L, K, q)\left\{h \left(\int_{t_{0}}^{x}(\sigma(x)-\sigma(s))\left(a(s) A^{K-1}(\sigma(s))^{q} \Delta s\right)\right.\right. \\
& +h^{*}\left(\int_{t_{0}}^{y}(\sigma(y)-\sigma(t))\left(b(t) B^{L-1}(\sigma(t))^{q} \Delta t\right)\right\}
\end{aligned}
$$

and

$$
\begin{aligned}
& \int_{t_{0}}^{x} \int_{t_{0}}^{y} \frac{A^{K}(\sigma(s)) B^{L}(\sigma(t))}{\left(\left|h\left(\sigma(s)-t_{0}\right)\right|^{\frac{1}{2 \beta}}+\left|h^{*}\left(\sigma(t)-t_{0}\right)\right|^{\frac{1}{2 \beta}}\right)^{\frac{2 \alpha}{p}} \Delta s \Delta t} \\
& \leqslant C_{2}(L, K, p)\left\{h\left(\int_{t_{0}}^{x}(\sigma(x)-\sigma(s))\left(A^{K-1}(\sigma(s)) a(s)\right)^{q} \Delta s\right)\right. \\
& \left.+h^{*}\left(\int_{t_{0}}^{y}(\sigma(y)-\sigma(t))\left(B^{L-1}(\sigma(t)) b(t)\right)^{q} \Delta t\right)\right\}^{\frac{1}{q}} .
\end{aligned}
$$

Proof. Using the Fenchel-Young inequality (5) in (11) and (12). This proves the claim.

Theorem 4. Assuming the time scale $\mathbb{T}$ with $s, t, t_{0}, x, y \in \mathbb{T}, A(s)$ and $B(t)$ are defined as in Theorem 2. Suppose $f(\check{\tau}) \geq 0$ and $g(\eta) \geq 0$ are right-dense continuous functions on $\left[t_{0}, x\right]_{\mathbb{T}}$ and $\left[t_{0}, y\right]_{\mathbb{T}}$ respectively. Suppose that $\breve{\Phi} \geq 0$ and $\breve{\Psi} \geq 0$ are convex, and submultiplicative functions on $[0, \infty)$. Furthermore assume that

$$
F(s):=\int_{t_{0}}^{s} f(\check{\tau}) \Delta \check{\tau} \text {, and } G(t):=\int_{t_{0}}^{t} g(\eta) \Delta \eta,
$$


then for $\sigma(s) \in\left[t_{0}, x\right]_{\mathbb{T}}$ and $\sigma(t) \in\left[t_{0}, y\right]_{\mathbb{T}}$, we have that

$$
\begin{aligned}
& \int_{t_{0}}^{x} \int_{t_{0}}^{y} \frac{\breve{\Phi}\left(A^{\sigma}(s)\right) \breve{\Psi}\left(B^{\sigma}(t)\right)}{\left(\left|h\left(\sigma(s)-t_{0}\right)\right|^{\frac{1}{2 \beta}}+\left|h^{*}\left(\sigma(t)-t_{0}\right)\right|^{\frac{1}{2 \beta}}\right)^{\frac{2 \alpha}{p}}} \Delta s \Delta t \\
& \leqslant M_{1}(p)\left(\int_{t_{0}}^{x}(\sigma(x)-\sigma(s))\left(f(s) \breve{\Phi}\left(\frac{a(s)}{f(s)}\right)\right)^{q} \Delta s\right)^{\frac{1}{q}} \\
& \times\left(\int_{t_{0}}^{y}(\sigma(y)-\sigma(t))\left(g(t) \breve{\Psi}\left(\frac{b(t)}{g(t)}\right)\right)^{q} \Delta t\right)^{\frac{1}{q}}
\end{aligned}
$$

where

$$
M_{1}(p)=\left\{\int_{t_{0}}^{x}\left(\frac{\breve{\Phi}\left(F^{\sigma}(s)\right)}{F^{\sigma}(s)}\right)^{p} \Delta s\right\}^{\frac{1}{p}}\left\{\int_{t_{0}}^{y}\left(\frac{\breve{\Psi}\left(G^{\sigma}(t)\right)}{G^{\sigma}(t)}\right)^{p} \Delta t\right\}^{\frac{1}{p}}
$$

Proof. From the properties of $\breve{\Phi}$ and using (2), we obtain

$$
\begin{aligned}
\breve{\Phi}\left(A^{\sigma}(s)\right) & =\breve{\Phi}\left(\frac{F(\sigma(s)) \int_{t_{0}}^{\sigma(s)} f(\check{\tau}) \frac{a(\check{\tau})}{f(\check{\tau})} \Delta \check{\tau}}{\int_{t_{0}}^{\sigma(s)} f(\check{\tau}) \Delta \check{\tau}}\right) \\
& \leqslant \breve{\Phi}\left(F(\sigma(s)) \breve{\Phi}\left(\frac{\int_{t_{0}}^{\sigma(s)} f(\check{\tau}) \frac{a(\check{\tau})}{f(\check{\tau})} \Delta \check{\tau}}{\int_{t_{0}}^{\sigma(s)} f(\check{\tau}) \Delta \check{\tau}}\right)\right. \\
& \leqslant \frac{\breve{\Phi}(F(\sigma(s))}{F(\sigma(s))} \int_{t_{0}}^{\sigma(s)} f(\check{\tau}) \breve{\Phi}\left(\frac{a(\check{\tau})}{f(\check{\tau})}\right) \Delta \check{\tau} .
\end{aligned}
$$

Using (1) in (29), we see that

$$
\breve{\Phi}\left(A^{\sigma}(s)\right) \leqslant \frac{\breve{\Phi}\left(F^{\sigma}(s)\right)}{F^{\sigma}(s)}\left(\sigma(s)-t_{0}\right)^{\frac{1}{p}}\left(\int_{t_{0}}^{\sigma(s)}\left(f(\check{\tau}) \breve{\Phi}\left[\frac{a(\check{\tau})}{f(\check{\tau})}\right]\right)^{q} \Delta \check{\tau}\right)^{\frac{1}{q}} .
$$

In addition, from the convexity and submultiplicative property of $\breve{\Psi}$, we get by using (2) and (1):

$$
\breve{\Psi}\left(B^{\sigma}(t)\right) \leqslant \frac{\breve{\Psi}\left(G^{\sigma}(t)\right)}{G^{\sigma}(t)}\left(\sigma(t)-t_{0}\right)^{\frac{1}{p}}\left(\int_{t_{0}}^{\sigma(t)}\left(g(\eta) \breve{\Psi}\left[\frac{b(\eta)}{g(\eta)}\right]\right)^{q} \Delta \eta\right)^{\frac{1}{q}} .
$$

From (30) and (31), we have

$$
\begin{aligned}
\breve{\Phi}\left(A^{\sigma}(s)\right) \breve{\Psi}\left(B^{\sigma}(t)\right) \leqslant & \left(\sigma(s)-t_{0}\right)^{\frac{1}{p}}\left(\sigma(t)-t_{0}\right)^{\frac{1}{p}}\left(\frac{\breve{\Phi}\left(F^{\sigma}(s)\right)}{F^{\sigma}(s)}\left(\int_{t_{0}}^{\sigma(s)}\left(f(\check{\tau}) \breve{\Phi}\left[\frac{a(\check{\tau})}{f(\check{\tau})}\right]\right)^{q} \Delta \check{\tau}\right)^{\frac{1}{q}}\right. \\
& \times\left(\frac{\breve{\Psi}\left(G^{\sigma}(t)\right)}{G^{\sigma}(t)}\left(\int_{t_{0}}^{\sigma(t)}\left(g(\eta) \breve{\Psi}\left[\frac{b(\eta)}{g(\eta)}\right]\right)^{q} \Delta \eta\right)^{\frac{1}{q}}\right.
\end{aligned}
$$

Using (5) on $\left(\sigma(s)-t_{0}\right)^{\frac{1}{p}}\left(\sigma(t)-t_{0}\right)^{\frac{1}{p}}$ gives:

$$
\begin{aligned}
\breve{\Phi}\left(A^{\sigma}(s)\right) \breve{\Psi}\left(B^{\sigma}(t)\right) \leqslant & \left(h\left(\sigma(s)-t_{0}\right)+h^{*}\left(\sigma(t)-t_{0}\right)\right)^{\frac{1}{p}}\left(\frac{\breve{\Phi}\left(F^{\sigma}(s)\right)}{F^{\sigma}(s)}\left(\int_{t_{0}}^{\sigma(s)}\left(f(\check{\tau}) \breve{\Phi}\left[\frac{a(\check{\tau})}{f(\check{\tau})}\right]\right)^{q} \Delta \check{\tau}\right)^{\frac{1}{q}}\right. \\
& \times\left(\frac{\breve{\Psi}\left(G^{\sigma}(t)\right)}{G^{\sigma}(t)}\left(\int_{t_{0}}^{\sigma(t)}\left(g(\eta) \breve{\Psi}\left[\frac{b(\eta)}{g(\eta)}\right]\right)^{q} \Delta \eta\right)^{\frac{1}{q}} .\right.
\end{aligned}
$$


Applying Lemma 6 on the right hand side of (33), we see that

$$
\begin{aligned}
& \breve{\Phi}\left(A^{\sigma}(s)\right) \breve{\Psi}\left(B^{\sigma}(t)\right) \leqslant\left(\mid h\left(\sigma(s)-\left.t_{0}\right|^{\frac{1}{2 \beta}}+\left|h^{*}\left(\sigma(t)-t_{0}\right)\right|^{\frac{1}{2 \beta}}\right)^{\frac{2 \alpha}{p}}\right. \\
& \times\left(\frac{\breve{\Phi}\left(F^{\sigma}(s)\right)}{F^{\sigma}(s)}\left(\int_{t_{0}}^{\sigma(s)}\left(f(\check{\tau}) \breve{\Phi}\left[\frac{a(\check{\tau})}{f(\check{\tau})}\right]\right)^{q} \Delta \check{\tau}\right)^{\frac{1}{q}}\right. \\
& \times\left(\frac{\breve{\Psi}\left(G^{\sigma}(t)\right)}{G^{\sigma}(t)}\left(\int_{t_{0}}^{\sigma(t)}\left(g(\eta) \breve{\Psi}\left[\frac{b(\eta)}{g(\eta)}\right]\right)^{q} \Delta \eta\right)^{\frac{1}{q}} .\right.
\end{aligned}
$$

From (34), we have

$$
\begin{gathered}
\frac{\breve{\Phi}\left(A^{\sigma}(s)\right) \breve{\Psi}\left(B^{\sigma}(t)\right)}{\left(\mid h\left(\sigma(s)-\left.t_{0}\right|^{\frac{1}{2 \beta}}+\left|h^{*}\left(\sigma(t)-t_{0}\right)\right|^{\frac{1}{2 \beta}}\right)^{\frac{2 \alpha}{p}}\right.} \leqslant\left(\frac{\breve{\Phi}\left(F^{\sigma}(s)\right)}{F^{\sigma}(s)}\left(\int_{t_{0}}^{\sigma(s)}\left(f(\check{\tau}) \breve{\Phi}\left[\frac{a(\check{\tau})}{f(\check{\tau})}\right]\right)^{q} \Delta \check{\tau}\right)^{\frac{1}{q}}\right. \\
\times\left(\frac{\breve{\Psi}\left(G^{\sigma}(t)\right)}{G^{\sigma}(t)}\left(\int_{t_{0}}^{\sigma(t)}\left(g(\eta) \breve{\Psi}\left[\frac{b(\eta)}{g(\eta)}\right]\right)^{q} \Delta \eta\right)^{\frac{1}{q}} .\right.
\end{gathered}
$$

From (35), we obtain

$$
\begin{aligned}
\int_{t_{0}}^{x} \int_{t_{0}}^{y} \frac{\breve{\Phi}\left(A^{\sigma}(s)\right) \breve{\Psi}\left(B^{\sigma}(t)\right)}{\left(\mid h\left(\sigma(s)-\left.t_{0}\right|^{\frac{1}{2 \beta}}+\left|h^{*}\left(\sigma(t)-t_{0}\right)\right|^{\frac{1}{2 \beta}}\right)^{\frac{2 \alpha}{p}}\right.} \Delta s \Delta t \\
\leqslant \int_{t_{0}}^{x}\left(\frac{\breve{\Phi}\left(F^{\sigma}(s)\right)}{F^{\sigma}(s)}\left(\int_{t_{0}}^{\sigma(s)}\left(f(\check{\tau}) \breve{\Phi}\left[\frac{a(\check{\tau})}{f(\check{\tau})}\right]\right)^{q} \Delta \check{\tau}\right)^{\frac{1}{q}} \Delta s\right. \\
\quad \times \int_{t_{0}}^{y}\left(\frac{\breve{\Psi}\left(G^{\sigma}(t)\right)}{G^{\sigma}(t)}\left(\int_{t_{0}}^{\sigma(t)}\left(g(\eta) \breve{\Psi}\left[\frac{b(\eta)}{g(\eta)}\right]\right)^{q} \Delta \eta\right)^{\frac{1}{q}} \Delta t .\right.
\end{aligned}
$$

From (36), by using (1), we have

$$
\begin{gathered}
\int_{t_{0}}^{x} \int_{t_{0}}^{y} \frac{\breve{\Phi}\left(A^{\sigma}(s)\right) \breve{\Psi}\left(B^{\sigma}(t)\right)}{\left(\mid h\left(\sigma(s)-\left.t_{0}\right|^{\frac{1}{2 \beta}}+\left|h^{*}\left(\sigma(t)-t_{0}\right)\right|^{\frac{1}{2 \beta}}\right)^{\frac{2 \alpha}{p}}\right.} \Delta s \Delta t \\
\leqslant\left\{\int_{t_{0}}^{x}\left(\frac{\breve{\Phi}\left(F^{\sigma}(s)\right)}{F^{\sigma}(s)}\right)^{p} \Delta s\right\}^{\frac{1}{p}}\left(\int_{t_{0}}^{x} \int_{t_{0}}^{\sigma(s)}\left(f(\check{\tau}) \breve{\Phi}\left[\frac{a(\check{\tau})}{f(\check{\tau})}\right]\right)^{q} \Delta \check{\tau} \Delta s\right)^{\frac{1}{q}} \\
\times\left\{\int_{t_{0}}^{y}\left(\frac{\breve{\Psi}\left(G^{\sigma}(t)\right)}{G^{\sigma}(t)}\right)^{p} \Delta t\right\}^{\frac{1}{p}}\left(\int_{t_{0}}^{y} \int_{t_{0}}^{\sigma(t)}\left(g(\eta) \breve{\Psi}\left[\frac{b(\eta)}{g(\eta)}\right]\right)^{q} \Delta \eta \Delta t\right)^{\frac{1}{q}} .
\end{gathered}
$$

From (37), by using (5), we obtain

$$
\begin{aligned}
& \int_{t_{0}}^{x} \int_{t_{0}}^{y} \frac{\breve{\Phi}\left(A^{\sigma}(s)\right) \breve{\Psi}\left(B^{\sigma}(t)\right)}{\left(\mid h\left(\sigma(s)-\left.t_{0}\right|^{\frac{1}{2 \beta}}+\left|h^{*}\left(\sigma(t)-t_{0}\right)\right|^{\frac{1}{2 \beta}}\right)^{\frac{2 \alpha}{p}}\right.} \Delta s \Delta t \\
& \leqslant M_{1}(p)\left(\int_{t_{0}}^{x}(x-\sigma(s))\left(f(s) \breve{\Phi}\left[\frac{a(s)}{f(s)}\right]\right)^{q} \Delta s\right)^{\frac{1}{q}} \\
& \times\left(\int_{t_{0}}^{y}(y-\sigma(t))\left(g(t) \breve{\Psi}\left[\frac{b(t)}{g(t)}\right]\right)^{q} \Delta t\right)^{\frac{1}{q}} .
\end{aligned}
$$


By using the facts $\sigma(x) \geqslant x$ and $\sigma(y) \geqslant y$, we obtain

$$
\begin{aligned}
& \int_{t_{0}}^{x} \int_{t_{0}}^{y} \frac{\breve{\Phi}\left(A^{\sigma}(s)\right) \breve{\Psi}\left(B^{\sigma}(t)\right)}{\left(\mid h\left(\sigma(s)-\left.t_{0}\right|^{\frac{1}{2 \beta}}+\left|h^{*}\left(\sigma(t)-t_{0}\right)\right|^{\frac{1}{2 \beta}}\right)^{\frac{2 \alpha}{p}}\right.} \Delta s \Delta t \\
& \leqslant M_{1}(p)\left(\int_{t_{0}}^{x}(\sigma(x)-\sigma(s))\left(f(s) \breve{\Phi}\left[\frac{a(s)}{f(s)}\right]\right)^{q} \Delta s\right)^{\frac{1}{q}} \\
& \times\left(\int_{t_{0}}^{y}(\sigma(y)-\sigma(t))\left(g(t) \breve{\Psi}\left[\frac{b(t)}{g(t)}\right]\right)^{q} \Delta t\right)^{\frac{1}{q}}
\end{aligned}
$$

where

$$
M_{1}(p)=\left\{\int_{t_{0}}^{x}\left(\frac{\breve{\Phi}\left(F^{\sigma}(s)\right)}{F^{\sigma}(s)}\right)^{p} \Delta s\right\}^{\frac{1}{p}}\left\{\int_{t_{0}}^{y}\left(\frac{\breve{\Psi}\left(G^{\sigma}(t)\right)}{G^{\sigma}(t)}\right)^{p} \Delta t\right\}^{\frac{1}{p}} .
$$

This completes the proof.

In Theorem 4 , taking $\mathbb{T}=\mathbb{R}$, we have (1) and the result:

Corollary 5. Assume that $a(s) \geq 0, b(t) \geq 0, f(\check{\tau}) \geq 0$ and $g(\eta) \geq 0$, we define

$$
A(s):=\int_{0}^{s} a(\eta) d \eta, \quad B(t):=\int_{0}^{t} b(\eta) d \eta, \quad F(s):=\int_{0}^{s} f(\check{\tau}) d \check{\tau}, \text { and } G(t):=\int_{0}^{t} g(\eta) d \eta \text {. }
$$

Then

$$
\begin{aligned}
\int_{0}^{x} \int_{0}^{y} \frac{\breve{\Phi}(A(s) \breve{\Psi}(B(t))}{\left(|h(s)|^{\frac{1}{2 \beta}}+\left|h^{*}(t)\right|^{\frac{1}{2 \beta}}\right)^{\frac{2 \alpha}{p}}} d s d t & \leqslant M_{2}(p)\left(\int_{0}^{x}(x-s)\left(f(s) \breve{\Phi}\left(\frac{a(s)}{f(s)}\right)\right)^{q} d s\right)^{\frac{1}{q}} \\
& \times\left(\int_{0}^{y}(y-t)\left(g(t) \breve{\Psi}\left(\frac{b(t)}{g(t)}\right)\right)^{q} d t\right)^{\frac{1}{q}}
\end{aligned}
$$

where

$$
M_{2}(p)=\left\{\int_{0}^{x}\left(\frac{\breve{\Phi}(F(s))}{F(s)}\right)^{p} d s\right\}^{\frac{1}{p}}\left\{\int_{0}^{y}\left(\frac{\breve{\Psi}(G(t))}{G(t)}\right)^{p} d t\right\}^{\frac{1}{p}} .
$$

In Theorem 4 , taking $\mathbb{T}=\mathbb{Z}$, gives (2) and the result:

Corollary 6. Assume that $a(n) \geq 0, b(m) \geq 0, f(n) \geq 0, g(m) \geq 0$ are sequences of real numbers. Define

$$
A(n)=\sum_{s=0}^{n} a(s), B(m)=\sum_{k=0}^{m} b(k), F(n)=\sum_{s=0}^{n} f(s) \text { and } G(m)=\sum_{k=0}^{m} g(k) .
$$

Then

$$
\begin{aligned}
\sum_{n=1}^{N} \sum_{m=1}^{M} \frac{\breve{\Phi}(A(n)) \breve{\Psi}(B(m))}{\left(|h(n+1)|^{\frac{1}{2 \beta}}+\left|h^{*}(m+1)\right|^{\frac{1}{2 \beta}}\right)^{\frac{2 \alpha}{p}}} \leqslant & M_{3}(p)\left\{\sum_{n=1}^{N}(N+1-(n+1))\left(f(n) \breve{\Phi}\left[\frac{a(n)}{f(n)}\right]\right)^{q}\right\}^{\frac{1}{q}} \\
& \times\left\{\sum_{m=1}^{M}(M+1-(m+1))\left(g(m) \breve{\Psi}\left[\frac{b(m)}{g(m)}\right]\right)^{q}\right\}^{\frac{1}{q}}
\end{aligned}
$$


where

$$
M_{3}(p)=\left\{\sum_{n=1}^{N}\left(\frac{\breve{\Phi}(F(n)}{F(n)}\right)^{p}\right\}^{\frac{1}{p}}\left\{\sum_{m=1}^{M}\left(\frac{\breve{\Psi}(G(m)}{G(m)}\right)^{p}\right\}^{\frac{1}{p}}
$$

Remark 2. In Corollary 6, if $p=q=2$ we get the result due to Hamiaz and Abuelela ([1], Theorem 5).

Corollary 7. Under the hypotheses of Theorem 4 the following inequality hold:

$$
\begin{aligned}
& \int_{t_{0}}^{x} \int_{t_{0}}^{y} \frac{\breve{\Phi}\left(A^{\sigma}(s)\right) \breve{\Psi}\left(B^{\sigma}(t)\right)}{\left(\left|h\left(\sigma(s)-t_{0}\right)\right|^{\frac{1}{2 \beta}}+\left|h^{*}\left(\sigma(t)-t_{0}\right)\right|^{\frac{1}{2 \beta}}\right)^{\frac{2 \alpha}{p}} \Delta s \Delta t} \\
& \leqslant M_{1}(p)\left[h\left(\int_{t_{0}}^{x}(\sigma(x)-\sigma(s))\left(f(s) \breve{\Phi}\left(\frac{a(s)}{f(s)}\right)\right)^{q} \Delta s\right)\right. \\
& \left.+h^{*}\left(\int_{t_{0}}^{y}(\sigma(y)-\sigma(t))\left(g(t) \breve{\Psi}\left(\frac{b(t)}{g(t)}\right)\right)^{q} \Delta t\right)\right]^{\frac{1}{q}} .
\end{aligned}
$$

Proof. Using (5) in (28). This proves our claim.

Lemma 7. With hypotheses of Theorem 4, we get:

$$
\begin{aligned}
& \int_{t_{0}}^{x} \int_{t_{0}}^{y} \frac{\breve{\Phi}\left(A^{\sigma}(s)\right)^{2} \breve{\Psi}\left(B^{\sigma}(t)\right)^{2}}{\left(h\left(\sigma(s)-t_{0}\right)+h^{*}\left(\sigma(t)-t_{0}\right)\right)} \Delta s \Delta t \\
& \leqslant M_{4}\left\{\int_{t_{0}}^{x}(\sigma(x)-\sigma(s))\left(f(s) \breve{\Phi}\left[\frac{a(s)}{f(s)}\right]\right)^{4} \Delta s\right\}^{\frac{1}{2}}\left\{\int_{t_{0}}^{y}(\sigma(t)-\sigma(t))\left(g(t) \breve{\Psi}\left[\frac{b(t)}{g(t)}\right]\right)^{4} \Delta t\right\}^{\frac{1}{2}}(38)
\end{aligned}
$$

where

$$
M_{4}=\left\{\int_{t_{0}}^{x}\left(\frac{\breve{\Phi}\left(F^{\sigma}(s)\right)^{4}}{\left(F^{\sigma}(s)\right)^{4}}\right)\left(\sigma(s)-t_{0}\right) \Delta s\right\}^{\frac{1}{2}}\left\{\int_{t_{0}}^{y}\left(\frac{\breve{\Psi}\left(G^{\sigma}(t)\right)^{4}}{\left(G^{\sigma}(t)\right)^{4}}\right)\left(\sigma(t)-t_{0}\right) \Delta t\right\}^{\frac{1}{2}} .
$$

Proof. From (30) and (31) and by using Fenchel-Young inequality with $p=q=2$ we have

$$
\begin{aligned}
& \breve{\Phi}\left(A^{\sigma}(s)\right)^{2} \breve{\Psi}\left(B^{\sigma}(t)\right)^{2} \\
& \leqslant\left(h\left(\sigma(s)-t_{0}\right)+h^{*}\left(\sigma(t)-t_{0}\right)\right)\left(\frac{\breve{\Phi}\left(F^{\sigma}(s)\right)^{2}}{\left(F^{\sigma}(s)\right)^{2}}\left(\int_{t_{0}}^{\sigma(s)}\left(f(\check{\tau}) \breve{\Phi}\left[\frac{a(\check{\tau})}{f(\check{\tau})}\right]\right)^{2} \Delta \check{\tau}\right)\right. \\
& \times\left(\frac{\breve{\Psi}\left(G^{\sigma}(t)\right)^{2}}{\left(G^{\sigma}(t)\right)^{2}}\left(\int_{t_{0}}^{\sigma(t)}\left(g(\eta) \breve{\Psi}\left[\frac{b(\eta)}{g(\eta)}\right]\right)^{2} \Delta \eta\right) .\right.
\end{aligned}
$$


From (40), by using (1) with $p=q=2$, we obtain

$$
\begin{aligned}
& \int_{t_{0}}^{x} \int_{t_{0}}^{y} \frac{\breve{\Phi}\left(A^{\sigma}(s)\right)^{2} \breve{\Psi}\left(B^{\sigma}(t)\right)^{2}}{\left(h\left(\sigma(s)-t_{0}\right)+h^{*}\left(\sigma(t)-t_{0}\right)\right)} \Delta s \Delta t \\
& \leqslant \int_{t_{0}}^{x}\left(\frac{\breve{\Phi}\left(F^{\sigma}(s)\right)^{2}}{\left(F^{\sigma}(s)\right)^{2}}\left(\int_{t_{0}}^{\sigma(s)}\left(f(\check{\tau}) \breve{\Phi}\left[\frac{a(\check{\tau})}{f(\check{\tau})}\right]\right)^{2} \Delta \check{\tau}\right) \Delta s\right. \\
& \times \int_{t_{0}}^{y} \frac{\breve{\Psi}\left(G^{\sigma}(t)\right)^{2}}{\left(G^{\sigma}(t)\right)^{2}}\left(\int_{t_{0}}^{\sigma(t)}\left(g(\eta) \breve{\Psi}\left[\frac{b(\eta)}{g(\eta)}\right]\right)^{2} \Delta \eta\right) \Delta t \\
& \leqslant \int_{t_{0}}^{x}\left(\frac{\breve{\Phi}\left(F^{\sigma}(s)\right)^{2}}{\left(F^{\sigma}(s)\right)^{2}}\left(\sigma(s)-t_{0}\right)^{\frac{1}{2}}\left(\int_{t_{0}}^{\sigma(s)}\left(f(\check{\tau}) \breve{\Phi}\left[\frac{a(\check{\tau})}{f(\check{\tau})}\right]\right)^{4} \Delta \check{\tau}\right)^{\frac{1}{2}}\right) \Delta s \\
& \times \int_{t_{0}}^{y} \frac{\breve{\Psi}\left(G^{\sigma}(t)\right)^{2}}{\left(G^{\sigma}(t)\right)^{2}}\left(\sigma(t)-t_{0}\right)^{\frac{1}{2}}\left(\int_{t_{0}}^{\sigma(t)}\left(g(\eta) \breve{\Psi}\left[\frac{b(\eta)}{g(\eta)}\right]\right)^{4} \Delta \eta\right)^{\frac{1}{2}} \Delta t \\
& \leqslant\left\{\int_{t_{0}}^{x}\left(\frac{\breve{\Phi}\left(F^{\sigma}(s)\right)^{4}}{\left(F^{\sigma}(s)\right)^{4}}\right)\left(\sigma(s)-t_{0}\right) \Delta s\right\}^{\frac{1}{2}}\left\{\int_{t_{0}}^{x}\left(\int_{t_{0}}^{\sigma(s)}\left(f(\check{\tau}) \breve{\Phi}\left[\frac{a(\check{\tau})}{f(\check{\tau})}\right]\right)^{4} \Delta \check{\tau}\right) \Delta s\right\}^{\frac{1}{2}} \\
& \times\left\{\int_{t_{0}}^{y}\left(\frac{\breve{\Psi}\left(G^{\sigma}(t)\right)^{4}}{\left(G^{\sigma}(t)\right)^{4}}\right)\left(\sigma(t)-t_{0}\right) \Delta t\right\}^{\frac{1}{2}}\left\{\int_{t_{0}}^{y}\left(\int_{t_{0}}^{\sigma(t)}\left(g(\eta) \breve{\Psi}\left[\frac{b(\eta)}{g(\eta)}\right]\right)^{4} \Delta \eta\right) \Delta t\right\}^{\frac{1}{2}} .
\end{aligned}
$$

Applying (5) on (41), we obtain

$$
\begin{aligned}
& \int_{t_{0}}^{x} \int_{t_{0}}^{y} \frac{\breve{\Phi}\left(A^{\sigma}(s)\right)^{2} \breve{\Psi}\left(B^{\sigma}(t)\right)^{2}}{\left(h\left(\sigma(s)-t_{0}\right)+h^{*}\left(\sigma(t)-t_{0}\right)\right)} \Delta s \Delta t \\
& \leqslant\left\{\int_{t_{0}}^{x}\left(\frac{\breve{\Phi}\left(F^{\sigma}(s)\right)^{4}}{\left(F^{\sigma}(s)\right)^{4}}\right)\left(\sigma(s)-t_{0}\right) \Delta s\right\}^{\frac{1}{2}}\left\{\int_{t_{0}}^{x}(x-\sigma(s))\left(f(s) \breve{\Phi}\left[\frac{a(s)}{f(s)}\right]\right)^{4} \Delta s\right\}^{\frac{1}{2}} \\
& \times\left\{\int_{t_{0}}^{y}\left(\frac{\breve{\Psi}\left(G^{\sigma}(t)\right)^{4}}{\left(G^{\sigma}(t)\right)^{4}}\right)\left(\sigma(t)-t_{0}\right) \Delta t\right\}^{\frac{1}{2}}\left\{\int_{t_{0}}^{y}(t-\sigma(t))\left(g(t) \breve{\Psi}\left[\frac{b(t)}{g(t)}\right]\right)^{4} \Delta t\right\}^{\frac{1}{2}} \\
& =M_{4}\left\{\int_{t_{0}}^{x}(x-\sigma(s))\left(f(s) \breve{\Phi}\left[\frac{a(s)}{f(s)}\right]\right)^{4} \Delta s\right\}^{\frac{1}{2}}\left\{\int_{t_{0}}^{y}(t-\sigma(t))\left(g(t) \breve{\Psi}\left[\frac{b(t)}{g(t)}\right]\right)^{4} \Delta t\right\}^{\frac{1}{2}} .
\end{aligned}
$$

Since $\sigma(x) \geqslant x$ and $\sigma(y) \geqslant y$, from the last inequality above, we have

$$
\begin{aligned}
& \int_{t_{0}}^{x} \int_{t_{0}}^{y} \frac{\breve{\Phi}\left(A^{\sigma}(s)\right)^{2} \breve{\Psi}\left(B^{\sigma}(t)\right)^{2}}{\left(h\left(\sigma(s)-t_{0}\right)+h^{*}\left(\sigma(t)-t_{0}\right)\right)} \Delta s \Delta t \\
& \leqslant M_{4}\left\{\int_{t_{0}}^{x}(\sigma(x)-\sigma(s))\left(f(s) \breve{\Phi}\left[\frac{a(s)}{f(s)}\right]\right)^{4} \Delta s\right\}^{\frac{1}{2}}\left\{\int_{t_{0}}^{y}(\sigma(t)-\sigma(t))\left(g(t) \breve{\Psi}\left[\frac{b(t)}{g(t)}\right]\right)^{4} \Delta t\right\}^{\frac{1}{2}}
\end{aligned}
$$

where $M_{4}$ defined as in (39). This proves our claim.

Theorem 5. Assume the time scale $\mathbb{T}$ with $t, s, x_{0}, t_{0}, y \in \mathbb{T}$. Suppose that $b(\check{\tau}) \geq 0$ and $a(\check{\tau}) \geq 0$ are right-dense continuous functions on $\left[t_{0}, y\right]_{\mathbb{T}}$ and $\left[t_{0}, x\right]_{\mathbb{T}}$. Let $G, F, g, f, \breve{\Psi}$ and $\breve{\Phi}$ be as assumed in Theorem 4 . Furthermore assume that

$$
A(s):=\frac{1}{F(s)} \int_{t_{0}}^{s} a(\check{\tau}) f(\check{\tau}) \Delta \check{\tau} \text {, and } B(t):=\frac{1}{G(t)} \int_{t_{0}}^{t} b(\eta) g(\eta) \Delta \eta,
$$


then for $\sigma(s) \in\left[t_{0}, x\right]_{\mathbb{T}}$ and $\sigma(t) \in\left[t_{0}, y\right]_{\mathbb{T}}$, we have that

$$
\begin{gathered}
\int_{t_{0}}^{x} \int_{t_{0}}^{y} \frac{\breve{\Phi}\left(A^{\sigma}(s)\right) \breve{\Psi}\left(B^{\sigma}(t)\right) F^{\sigma}(s) G^{\sigma}(t)}{\left(\left|h\left(\sigma(s)-t_{0}\right)\right|^{\frac{1}{2 \beta}}+\left|h^{*}\left(\sigma(t)-t_{0}\right)\right|^{\frac{1}{2 \beta}}\right)^{\frac{2 \alpha}{p}} \Delta s \Delta t} \\
\leqslant M_{5}(p)\left(\int_{t_{0}}^{x}(\sigma(x)-\sigma(s))(f(s) \breve{\Phi}(a(s)))^{q} \Delta s\right)^{\frac{1}{q}} \\
\times\left(\int_{t_{0}}^{y}(\sigma(y)-\sigma(t))(g(t) \breve{\Psi}(b(t)))^{q} \Delta t\right)^{\frac{1}{q}}
\end{gathered}
$$

where

$$
M_{5}(p)=\left(x-t_{0}\right)^{\frac{1}{p}}\left(y-t_{0}\right)^{\frac{1}{p}} .
$$

Proof. From (42), we see that

$$
\breve{\Phi}\left(A^{\sigma}(s)\right)=\breve{\Phi}\left(\frac{1}{F^{\sigma}(s)} \int_{t_{0}}^{\sigma(s)} f(\check{\tau}) a(\check{\tau}) \Delta \check{\tau}\right) .
$$

Applying (1) on (45), we obtain

$$
\breve{\Phi}\left(A^{\sigma}(s)\right) \leqslant \frac{\left(\sigma(s)-t_{0}\right)^{\frac{1}{p}}}{F^{\sigma}(s)}\left(\int_{t_{0}}^{\sigma(s)}(f(\check{\tau}) \breve{\Phi}[a(\check{\tau})])^{q} \Delta \check{\tau}\right)^{\frac{1}{q}} .
$$

From (46), we get

$$
\breve{\Phi}\left(A^{\sigma}(s)\right) F^{\sigma}(s) \leqslant\left(\sigma(s)-t_{0}\right)^{\frac{1}{p}}\left(\int_{t_{0}}^{\sigma(s)}(f(\check{\tau}) \breve{\Phi}[a(\check{\tau})])^{q} \Delta \check{\tau}\right)^{\frac{1}{q}} .
$$

Similarly, we obtain

$$
\breve{\Psi}\left(B^{\sigma}(t)\right) G^{\sigma}(t) \leqslant\left(\sigma(t)-t_{0}\right)^{\frac{1}{p}}\left(\int_{t_{0}}^{\sigma(t)}(g(\eta) \breve{\Psi}[b(\eta)])^{q} \Delta \eta\right)^{\frac{1}{q}} .
$$

From (47) and (48), we observe that

$$
\begin{aligned}
& \breve{\Phi}\left(A^{\sigma}(s)\right) \breve{\Psi}\left(B^{\sigma}(t)\right) G^{\sigma}(t) F^{\sigma}(s) \leqslant\left(\sigma(s)-t_{0}\right)^{\frac{1}{p}}\left(\sigma(t)-t_{0}\right)^{\frac{1}{p}} \\
& \quad \times\left(\int_{t_{0}}^{\sigma(s)}(f(\check{\tau}) \breve{\Phi}[a(\check{\tau})])^{q} \Delta \check{\tau}\right)^{\frac{1}{q}}\left(\int_{t_{0}}^{\sigma(t)}(g(\eta) \breve{\Psi}[b(\eta)])^{q} \Delta \eta\right)^{\frac{1}{q}} .
\end{aligned}
$$

Applying the Lemma 3 on the term $\left(\sigma(s)-t_{0}\right)^{\frac{1}{p}}\left(\sigma(t)-t_{0}\right)^{\frac{1}{p}}$, gives:

$$
\begin{aligned}
\breve{\Phi}\left(A^{\sigma}(s)\right) \breve{\Psi}\left(B^{\sigma}(t)\right) G^{\sigma}(t) F^{\sigma}(s) & \leqslant\left(h\left(\sigma(s)-t_{0}\right)+h^{*}\left(\sigma(t)-t_{0}\right)\right)^{\frac{1}{p}}\left(\int_{t_{0}}^{\sigma(s)}(f(\check{\tau}) \breve{\Phi}[a(\check{\tau})])^{q} \Delta \check{\tau}\right)^{\frac{1}{q}} \\
& \times\left(\int_{t_{0}}^{\sigma(t)}(g(\eta) \breve{\Psi}[b(\eta)])^{q} \Delta \eta\right)^{\frac{1}{q}} .
\end{aligned}
$$


From 6 and (50), we obtain

$$
\begin{gathered}
\breve{\Phi}\left(A^{\sigma}(s)\right) \breve{\Psi}\left(B^{\sigma}(t)\right) G^{\sigma}(t) F^{\sigma}(s) \leqslant\left(\left|h\left(\sigma(s)-t_{0}\right)\right|^{\frac{1}{2 \beta}}+\left|h^{*}\left(\sigma(t)-t_{0}\right)\right|^{\frac{1}{2 \beta}}\right)^{\frac{2 \alpha}{p}} \\
\times\left(\int_{t_{0}}^{\sigma(s)}(f(\check{\tau}) \breve{\Phi}[a(\check{\tau})])^{q} \Delta \check{\tau}\right)^{\frac{1}{q}}\left(\int_{t_{0}}^{\sigma(t)}(g(\eta) \breve{\Psi}[b(\eta)])^{q} \Delta \eta\right)^{\frac{1}{q}} .
\end{gathered}
$$

Dividing both sides of (51) by $\left(\left|h\left(\sigma(s)-t_{0}\right)\right|^{\frac{1}{2 \beta}}+\left|h^{*}\left(\sigma(t)-t_{0}\right)\right|^{\frac{1}{2 \beta}}\right)^{\frac{2 \alpha}{p}}$, we get

$$
\begin{aligned}
\frac{\breve{\Phi}\left(A^{\sigma}(s)\right) \breve{\Psi}\left(B^{\sigma}(t)\right) G^{\sigma}(t) F^{\sigma}(s)}{\left(\left|h\left(\sigma(s)-t_{0}\right)\right|^{\frac{1}{2 \beta}}+\left|h^{*}\left(\sigma(t)-t_{0}\right)\right|^{\frac{1}{2 \beta}}\right)^{\frac{2 \alpha}{p}}} & \leqslant\left(\int_{t_{0}}^{\sigma(s)}(f(\check{\tau}) \breve{\Phi}[a(\check{\tau})])^{q} \Delta \check{\tau}\right)^{\frac{1}{q}} \\
& \times\left(\int_{t_{0}}^{\sigma(t)}(g(\eta) \breve{\Psi}[b(\eta)])^{q} \Delta \eta\right)^{\frac{1}{q}} .
\end{aligned}
$$

Taking delta-integral for (52), yields:

$$
\begin{aligned}
& \int_{t_{0}}^{x} \int_{t_{0}}^{y} \frac{\breve{\Phi}\left(A^{\sigma}(s)\right) \breve{\Psi}\left(B^{\sigma}(t)\right) G^{\sigma}(t) F^{\sigma}(s)}{\left(\left|h\left(\sigma(s)-t_{0}\right)\right|^{\frac{1}{2 \beta}}+\left|h^{*}\left(\sigma(t)-t_{0}\right)\right|^{\frac{1}{2 \beta}}\right)^{\frac{2 \alpha}{p}}} \Delta s \Delta t \\
& \leqslant\left(\int_{t_{0}}^{x}\left(\int_{t_{0}}^{\sigma(s)}(f(\check{\tau}) \breve{\Phi}[a(\check{\tau})])^{q} \Delta \check{\tau}\right)^{\frac{1}{q}} \Delta s\right)\left(\int_{t_{0}}^{y}\left(\int_{t_{0}}^{\sigma(t)}(g(\eta) \breve{\Psi}[b(\eta)])^{q} \Delta \eta\right)^{\frac{1}{q}} \Delta t\right) .
\end{aligned}
$$

Using (1) in (53), yield:

$$
\begin{aligned}
& \int_{t_{0}}^{x} \int_{t_{0}}^{y} \frac{\breve{\Phi}\left(A^{\sigma}(s)\right) \breve{\Psi}\left(B^{\sigma}(t)\right) G^{\sigma}(t) F^{\sigma}(s)}{\left(\left|h\left(\sigma(s)-t_{0}\right)\right|^{\frac{1}{2 \beta}}+\left|h^{*}\left(\sigma(t)-t_{0}\right)\right|^{\frac{1}{2 \beta}}\right)^{\frac{2 \alpha}{p}} \Delta s \Delta t} \\
& \leqslant\left(x-t_{0}\right)^{\frac{1}{p}}\left(y-t_{0}\right)^{\frac{1}{p}}\left(\int_{t_{0}}^{x}\left(\int_{t_{0}}^{\sigma(s)}(f(\check{\tau}) \breve{\Phi}[a(\check{\tau})])^{q} \Delta \check{\tau}\right) \Delta s\right)^{\frac{1}{q}} \\
& \times\left(\int_{t_{0}}^{y}\left(\int_{t_{0}}^{\sigma(t)}(g(\eta) \breve{\Psi}[b(\eta)])^{q} \Delta \eta\right) \Delta t\right)^{\frac{1}{q}} \\
& =M_{5}(p)\left(\int_{t_{0}}^{x}\left(\int_{t_{0}}^{\sigma(s)}(f(\check{\tau}) \breve{\Phi}[a(\check{\tau})])^{q} \Delta \check{\tau}\right) \Delta s\right)^{\frac{1}{q}} \\
& \times\left(\int_{t_{0}}^{y}\left(\int_{t_{0}}^{\sigma(t)}(g(\eta) \breve{\Psi}[b(\eta)])^{q} \Delta \eta\right) \Delta t\right)^{\frac{1}{q}},
\end{aligned}
$$


where $M_{5}$ defined as in (44). From (5) and (54), we get:

$$
\begin{aligned}
& \int_{t_{0}}^{x} \int_{t_{0}}^{y} \frac{\breve{\Phi}\left(A^{\sigma}(s)\right) \breve{\Psi}\left(B^{\sigma}(t)\right) G^{\sigma}(t) F^{\sigma}(s)}{\left(\left|h\left(\sigma(s)-t_{0}\right)\right|^{\frac{1}{2 \beta}}+\left|h^{*}\left(\sigma(t)-t_{0}\right)\right|^{\frac{1}{2 \beta}}\right)^{\frac{2 \alpha}{p}}} \Delta s \Delta t \\
& =M_{5}(p)\left(\int_{t_{0}}^{x}(x-\sigma(s))(f(s) \breve{\Phi}[a(s)])^{q} \Delta s\right)^{\frac{1}{q}} \\
& \times\left(\int_{t_{0}}^{y}(y-\sigma(t))(g(t) \breve{\Psi}[b(t)])^{q} \Delta t\right)^{\frac{1}{q}} .
\end{aligned}
$$

By using the fact $\sigma(x) \geqslant x$ and $\sigma(y) \geqslant y$, we obtain

$$
\begin{aligned}
& \int_{t_{0}}^{x} \int_{t_{0}}^{y} \frac{\breve{\Phi}\left(A^{\sigma}(s)\right) \breve{\Psi}\left(B^{\sigma}(t)\right) G^{\sigma}(t) F^{\sigma}(s)}{\left(\left|h\left(\sigma(s)-t_{0}\right)\right|^{\frac{1}{2 \beta}}+\left|h^{*}\left(\sigma(t)-t_{0}\right)\right|^{\frac{1}{2 \beta}}\right)^{\frac{2 \alpha}{p}}} \Delta s \Delta t \\
& =M_{5}(p)\left(\int_{t_{0}}^{x}(\sigma(x)-\sigma(s))(f(s) \breve{\Phi}[a(s)])^{q} \Delta s\right)^{\frac{1}{q}} \\
& \times\left(\int_{t_{0}}^{y}(\sigma(y)-\sigma(t))(g(t) \breve{\Psi}[b(t)])^{q} \Delta t\right)^{\frac{1}{q}} .
\end{aligned}
$$

This completes the proof.

Taking $\mathbb{T}=\mathbb{R}$ in Theorem 5 with relation (1), we have:

Corollary 8. Assume $g(t) \geq 0, b(t) \geq 0, f(s) \geq 0, a(s) \geq 0$. Define

$$
\begin{gathered}
A(s):=\frac{1}{F(s)} \int_{0}^{s} f(\check{\tau}) a(\check{\tau}) d \check{\tau} \text { and } B(t):=\frac{1}{G(t)} \int_{0}^{t} g(\check{\tau}) b(\check{\tau}) d \check{\tau}, \\
F(s):=\int_{0}^{s} f(\check{\tau}) d \check{\tau} \text { and } G(t):=\int_{0}^{t} g(\check{\tau}) d \check{\tau} .
\end{gathered}
$$

Then

$$
\begin{aligned}
\int_{0}^{x} \int_{0}^{y} \frac{\breve{\Phi}(A(s)) \breve{\Psi}(B(t)) F(s) G(t)}{\left(|h(s)|^{\frac{1}{2 \beta}}+\left|h^{*}(t)\right|^{\frac{1}{2 \beta}}\right)^{\frac{2 \alpha}{p}}} d s d t & \leqslant M_{6}(p)\left(\int_{0}^{x}(x-s)(f(s) \breve{\Phi}(a(s)))^{q} d s\right)^{\frac{1}{q}} . \\
& \times\left(\int_{0}^{y}(y-t)(g(t) \breve{\Psi}(b(t)))^{q} d t\right)^{\frac{1}{q}}
\end{aligned}
$$

where

$$
M_{6}(p)=(x)^{\frac{1}{p}}(y)^{\frac{1}{p}}
$$

Taking $\mathbb{T}=\mathbb{Z}$ in Theorem 5 with relation (2), gives:

Corollary 9. Assume $g(n) \geq 0, b(n) \geq 0, f(n) \geq 0, a(n) \geq 0$. Define

$$
A(n):=\frac{1}{F(n)} \sum_{s=0}^{n} f(s) a(s) \text { and } B(m):=\frac{1}{G(m)} \sum_{k=0}^{m} g(k) b(k) .
$$




$$
F(n):=\sum_{s=0}^{n} f(s) \text { and } G(m):=\sum_{k=0}^{m} g(k) \text {. }
$$

Then

$$
\begin{aligned}
\sum_{n=1}^{N} \sum_{m=1}^{M} \frac{\breve{\Phi}(A(n)) \breve{\Psi}(B(m)) F(n) G(m)}{\left(|h(n+1)|^{\frac{1}{2 \beta}}+\left|h^{*}(m+1)\right|^{\frac{1}{2 \beta}}\right)^{\frac{2 \alpha}{p}}} & \leqslant M_{7}(p)\left(\sum_{n=1}^{N}(N+1-(n+1))(f(n) \breve{\Phi}(a(n)))^{q}\right)^{\frac{1}{q}} . \\
& \times\left(\sum_{m=1}^{M}(M+1-(m+1))(g(m) \breve{\Psi}(b(m)))^{q}\right)^{\frac{1}{q}}
\end{aligned}
$$

where

$$
M_{7}(p)=(N M)^{\frac{1}{p}}
$$

Remark 3. In Corollary 9, if $p=q=2$ we get the result due to Hamiaz and Abuelela ([1], Theorem 7).

Corollary 10. With the hypotheses of Theorem 5, we get:

$$
\begin{aligned}
& \int_{t_{0}}^{x} \int_{t_{0}}^{y} \frac{\breve{\Phi}\left(A^{\sigma}(s)\right) \breve{\Psi}\left(B^{\sigma}(t)\right) F^{\sigma}(s) G^{\sigma}(t)}{\left(\left|h\left(\sigma(s)-t_{0}\right)\right|^{\frac{1}{2 \beta}}+\left|h^{*}\left(\sigma(t)-t_{0}\right)\right|^{\frac{1}{2 \beta}}\right)^{\frac{2 \alpha}{p}} \Delta s \Delta t} \\
& \leqslant M_{5}(p)\left\{h\left(\int_{t_{0}}^{x}(\sigma(x)-\sigma(s))(f(s) \breve{\Phi}(a(s)))^{q} \Delta s\right)\right. \\
& \left.+h^{*}\left(\int_{t_{0}}^{y}(\sigma(y)-\sigma(t))(g(t) \breve{\Psi}(b(t)))^{q} \Delta t\right)\right\}^{\frac{1}{q}} .
\end{aligned}
$$

where $M_{5}$ defined as in (44).

Proof. We apply the Fenchel-Young inequality (5) in (43). This completes the proof.

\section{Some Applications}

We can apply our inequalities to obtain different formulas of Hilbert-type inequalities by suggesting $h^{*}(y)$ and $h(x)$ by some functions:

In (12), as a special case, if we take $h(x)=\frac{x^{2}}{2}$, we have $h^{*}(x)=\frac{x^{2}}{2}$ see [12], we get

$$
\begin{aligned}
& \int_{t_{0}}^{x} \int_{t_{0}}^{y} \frac{A^{K}(\sigma(s)) B^{L}(\sigma(t))}{\left(\left|h\left(\sigma(s)-t_{0}\right)\right|^{\frac{1}{2 \beta}}+\left|h^{*}\left(\sigma(t)-t_{0}\right)\right|^{\frac{1}{2 \beta}}\right)^{\frac{2 \alpha}{p}}} \Delta s \Delta t \\
& =\int_{t_{0}}^{x} \int_{t_{0}}^{y} \frac{A^{K}(\sigma(s)) B^{L}(\sigma(t))}{\left.\left(\left(\sigma(s)-t_{0}\right)\right)^{\frac{1}{\beta}}+\left(\sigma(t)-t_{0}\right)^{\frac{1}{\beta}}\right)^{\frac{2 \alpha}{p}} \Delta s \Delta t} \\
& \leqslant\left(\frac{1}{2}\right)^{\frac{\alpha}{p \beta}} C_{2}(L, K, p)\left(\int_{t_{0}}^{x}(\sigma(x)-\sigma(s))\left(A^{K-1}(\sigma(s)) a(s)\right)^{q} \Delta s\right)^{\frac{1}{q}} \\
& \times\left(\int_{t_{0}}^{y}(\sigma(y)-\sigma(t))\left(B^{L-1}(\sigma(t)) b(t)\right)^{q} \Delta t\right)^{\frac{1}{q}},
\end{aligned}
$$


where $C_{2}(L, K, p)$ defined as in Theorem 2. Consequently, for $\alpha=\beta=1$, inequality (55) produces

$$
\begin{aligned}
& \int_{t_{0}}^{x} \int_{t_{0}}^{y} \frac{A^{K}(\sigma(s)) B^{L}(\sigma(t))}{\left.\left(\left(\sigma(s)-t_{0}\right)\right)+\left(\sigma(t)-t_{0}\right)\right)^{\frac{2}{p}}} \Delta s \Delta t \\
& \leqslant\left(\frac{1}{2}\right)^{\frac{1}{p}} C_{2}(L, K, p)\left(\int_{t_{0}}^{x}(\sigma(x)-\sigma(s))\left(A^{K-1}(\sigma(s)) a(s)\right)^{q} \Delta s\right)^{\frac{1}{q}} \\
& \quad \times\left(\int_{t_{0}}^{y}(\sigma(y)-\sigma(t))\left(B^{L-1}(\sigma(t)) b(t)\right)^{q} \Delta t\right)^{\frac{1}{q}} .
\end{aligned}
$$

On the other hand if we take $h(n)=\frac{n^{r}}{r}, r>1$, then $h^{*}(m)=\frac{m^{k}}{k}$ where $\frac{1}{r}+\frac{1}{k}=1$ and $n, m \mathbb{R}_{+}$, then (12) gives

$$
\begin{aligned}
& \int_{t_{0}}^{x} \int_{t_{0}}^{y} \frac{A^{K}(\sigma(s)) B^{L}(\sigma(t))}{\left(\left|h\left(\sigma(s)-t_{0}\right)\right|^{\frac{1}{2 \beta}}+\left|h^{*}\left(\sigma(t)-t_{0}\right)\right|^{\frac{1}{2 \beta}}\right)^{\frac{2 \alpha}{p}}} \Delta s \Delta t \\
& =\int_{t_{0}}^{x} \int_{t_{0}}^{y} \frac{A^{K}(\sigma(s)) B^{L}(\sigma(t))}{\left(\left(k\left(\sigma(s)-t_{0}\right)^{r}\right)^{\frac{1}{2 \beta}}+\left(r\left(\sigma(t)-t_{0}\right)^{k}\right)^{\frac{1}{2 \beta}}\right)^{\frac{2 \alpha}{p}} \Delta s \Delta t} \\
& \leqslant\left(\frac{1}{r k}\right)^{\frac{\alpha}{p \beta}} C_{2}(L, K, p)\left(\int_{t_{0}}^{x}(\sigma(x)-\sigma(s))\left(A^{K-1}(\sigma(s)) a(s)\right)^{q} \Delta s\right)^{\frac{1}{q}} \\
& \quad \times\left(\int_{t_{0}}^{y}(\sigma(y)-\sigma(t))\left(B^{L-1}(\sigma(t)) b(t)\right)^{q} \Delta t\right)^{\frac{1}{q}} .
\end{aligned}
$$

Clearly, when $\beta=\frac{1}{2 \alpha}$, the inequality (57) becomes

$$
\begin{aligned}
& \int_{t_{0}}^{x} \int_{t_{0}}^{y} \frac{A^{K}(\sigma(s)) B^{L}(\sigma(t))}{\left(\left(k\left(\sigma(s)-t_{0}\right)^{r}\right)^{\alpha}+\left(r\left(\sigma(t)-t_{0}\right)^{k}\right)^{\alpha}\right)^{\frac{2 \alpha}{p}}} \Delta s \Delta t \\
& \leqslant\left(\frac{1}{r k}\right)^{\frac{2 \alpha^{2}}{p}} C_{2}(L, K, p)\left(\int_{t_{0}}^{x}(\sigma(x)-\sigma(s))\left(A^{K-1}(\sigma(s)) a(s)\right)^{q} \Delta s\right)^{\frac{1}{q}} \\
& \times\left(\int_{t_{0}}^{y}(\sigma(y)-\sigma(t))\left(B^{L-1}(\sigma(t)) b(t)\right)^{q} \Delta t\right)^{\frac{1}{q}} .
\end{aligned}
$$

If $\beta=\alpha=1$. From (57), we get

$$
\begin{aligned}
& \int_{t_{0}}^{x} \int_{t_{0}}^{y} \frac{A^{K}(\sigma(s)) B^{L}(\sigma(t))}{\left(\left(k\left(\sigma(s)-t_{0}\right)^{r}\right)^{\frac{1}{2}}+\left(r\left(\sigma(t)-t_{0}\right)^{k}\right)^{\frac{1}{2}}\right)^{\frac{2}{p}}} \Delta s \Delta t \\
& \leqslant\left(\frac{1}{r k}\right)^{\frac{1}{p}} C_{2}(L, K, p)\left(\int_{t_{0}}^{x}(\sigma(x)-\sigma(s))\left(A^{K-1}(\sigma(s)) a(s)\right)^{q} \Delta s\right)^{\frac{1}{q}} \\
& \times\left(\int_{t_{0}}^{y}(\sigma(y)-\sigma(t))\left(B^{L-1}(\sigma(t)) b(t)\right)^{q} \Delta t\right)^{\frac{1}{q}} .
\end{aligned}
$$




\section{Conclusions}

In this paper, with the help of a Fenchel-Legendre transform, which is used in various problems involving symmetry, we generalized a number of Hilbert-type inequalities to a general time scale. Besides that, in order to obtain some new inequalities as special cases, we also extended our inequalities to discrete and continuous calculus. In the future, we can generalize these inequalities in a different way by using other mathematical tools.

Author Contributions: All authors have contributed equally. All authors have read and agreed to the published version of the manuscript.

Funding: The authors declare that they have received no funding from any funding body.

Conflicts of Interest: The authors declare that they have no competing interests.

\section{References}

1. Hamiaz, A.; Abuelela, W. Some new discrete Hilbert's inequalities involving Fenchel-Legendre transform. J. Inequal. Appl. 2020, 2020, 1-14. [CrossRef]

2. Hilger, S. Ein Maßkettenkalkül mit Anwendung auf Zentrumsmannigfaltigkeiten. Ph.D. Thesis, Universität Würzburg, Würzburg, Germany, 1988.

3. Hardy, G.; Littlewood, J.E.; Pólya, G. Inequalities; Cambridge University Press: London, UK; New York, NY, USA, 1952; Volume 2, pp. 151-218.

4. Zhong, J.; Yang, B. An extension of a multidimensional Hilbert-type inequality. J. Inequal. Appl. 2017, 2017, 1-12. [CrossRef]

5. Frazer, H. Note on Hilbert's inequality. J. Lond. Math. Soc. 1946, 1, 7-9. [CrossRef]

6. Chen, Q.; Yang, B. A survey on the study of Hilbert-type inequalities. J. Inequal. Appl. 2015, 2015, 302. [CrossRef]

7. Abuelela, W. On an inequality related to hilbert's with laplace transform. Int. J. Pure Appl. Math. 2015, 101, 87-94. [CrossRef]

8. Huang, Q.; Yang, B. A multiple Hilbert-type integral inequality with a non-homogeneous kernel. J. Inequal. Appl. 2013, 2013, 73. [CrossRef]

9. Kim, Y.H. An improvement of some inequalities similar to Hilbert's inequality. Int. J. Math. Math. Sci. 2001, 28, 211-221. [CrossRef]

10. Pachpatte, B. On some new inequalities similar to Hilbert's inequality. J. Math. Anal. Appl. 1998, 226, 166-179. [CrossRef]

11. Dong, Q.L.; Lu, Y.Y.; Yang, J.; He, S. Approximately solving multi-valued variational inequalities by using a projection and contraction algorithm. Numer. Algorithms 2017, 76, 799-812. [CrossRef]

12. Borwein, J.; Lewis, A.S. Convex Analysis and Nonlinear Optimization: Theory and Examples; Springer Science \& Business Media: Berlin, Germany, 2010.

13. Arnold, V.I. Lectures on Partial Differential Equations; Springer Science \& Business Media: Berlin, Germany, 2013.

14. Davies, G.; Petersen, G. On an inequality of Hardy's (II). Q. J. Math. 1964, 15, 35-40. [CrossRef]

15. Nemeth, J. Generalizations of hardy-littlewood inequality. Acta Sci. Math. 1971, 32, 295.

16. Pachpatte, B. A note on some series inequalities. Tamkang J. Math. 1995, 27, 77-80.

17. Pachpatte, B.G.; Talkies, N.A. A note on integral inequalities involving the product of two functions. J. Inequal. Pure Appl. Math. 2006, 7, 78.

18. Mitrinovic, D.S.; Vasic, P.M. Analytic Inequalities; Springer: Berlin, Germany, 1970; Volume 61.

19. Agarwal, R.; O'Regan, D.; Saker, S. Dynamic Inequalities on Time Scales; Springer: Cham, Swizerland, 2014; Volume 2014.

20. Saker, S.H.; El-Deeb, A.; Rezk, H.; Agarwal, R.P. On Hilbert's inequality on time scales. Appl. Anal. Discret. Math. 2017, 11, 399-423. [CrossRef]

21. Abdeldaim, A.; El-Deeb, A.A.; Agarwal, P.; El-Sennary, H.A. On some dynamic inequalities of Steffensen type on time scales. Math. Methods Appl. Sci. 2018, 41, 4737-4753. [CrossRef] 
22. Akin-Bohner, E.; Bohner, M.; Akin, F. Pachpatte inequalities on time scales. JIPAM. J. Inequal. Pure Appl. Math. 2005, 6, 23.

23. Bohner, M.; Matthews, T. The Grüss inequality on time scales. Commun. Math. Anal. 2007, 3, 1-8.

24. Bohner, M.; Matthews, T. Ostrowski inequalities on time scales. JIPAM. J. Inequal. Pure Appl. Math. 2008, $9,6,8$.

25. Dinu, C. Hermite-Hadamard inequality on time scales. J. Inequal. Appl. 2008, 287947. [CrossRef]

26. El-Deeb, A.A. On some generalizations of nonlinear dynamic inequalities on time scales and their applications. Appl. Anal. Discret. Math. 2019, 13, 10. [CrossRef]

27. El-Deeb, A.A.; Cheung, W.S. A variety of dynamic inequalities on time scales with retardation. J. Nonlinear Sci. Appl. 2018, 11, 1185-1206. [CrossRef]

28. El-Deeb, A.A.; El-Sennary, H.A.; Khan, Z.A. Some Steffensen-type dynamic inequalities on time scales. Adv. Differ. Equ. 2019, 2019, 246. [CrossRef]

29. El-Deeb, A.A.; Elsennary, H.A.; Cheung, W.S. Some reverse Hölder inequalities with Specht's ratio on time scales. J. Nonlinear Sci. Appl. 2018, 11, 444-455. [CrossRef]

30. El-Deeb, A.A.; Elsennary, H.A.; Nwaeze, E.R. Generalized weighted Ostrowski, trapezoid and Grüss type inequalities on time scales. Fasc. Math. 2018, 123-144. [CrossRef]

31. El-Deeb, A.A.; Xu, H.; Abdeldaim, A.; Wang, G. Some dynamic inequalities on time scales and their applications. Adv. Differ. Equ. 2019, 130. [CrossRef]

32. El-Deeb, A.A. Some Gronwall-Bellman type inequalities on time scales for Volterra-Fredholm dynamic integral equations. J. Egypt. Math. Soc. 2018, 26, 1-17. [CrossRef]

33. Hilscher, R. A time scales version of a Wirtinger-type inequality and applications. J. Comput. Appl. Math. 2002, 141, 219-226. [CrossRef]

34. Li, W.N. Some delay integral inequalities on time scales. Comput. Math. Appl. 2010, 59, 1929-1936. [CrossRef]

35. Řehák, P. Hardy inequality on time scales and its application to half-linear dynamic equations. J. Inequal. Appl. 2005, 495-507, doi:10.1155/JIA.2005.495. [CrossRef]

36. Tian, Y.; El-Deeb, A.A.; Meng, F. Some nonlinear delay Volterra-Fredholm type dynamic integral inequalities on time scales. Discret. Dyn. Nat. Soc. 2018, 5841985, doi:10.1155/2018/5841985. [CrossRef]

37. El-Deeb, A.; Khan, Z.A. Certain new dynamic nonlinear inequalities in two independent variables and applications. Bound. Value Probl. 2020, 2020, 31. [CrossRef]

38. El-Deeb, A.; El-Sennary, H.; Agarwal, P. Some opial-type inequalities with higher order delta derivatives on time scales. Rev. Real Acad. Cienc. Exactas Fís. Nat. Ser. A Mat. 2020, 114, 29. [CrossRef]

39. Abdeldaim, A.; El-Deeb, A.; Ahmed, R.G. On retarded nonlinear integral inequalities of gronwall and applications. J. Math. Inequal. 2019, 13, 1023-1038. [CrossRef]

40. El-Deeb, A.A.; Kh., F.M.; Ismail, G.A.F.; Khan, Z.A. Weighted dynamic inequalities of Opial-type on time scales. Adv. Differ. Equ. 2019, 2019, 393. [CrossRef]

41. Kh., F.M.; El-Deeb, A.A.; Abdeldaim, A.; Khan, Z.A. On some generalizations of dynamic Opial-type inequalities on time scales. Adv. Differ. Equ. 2019, 2019, 1-14.

42. Abdeldaim, A.; El-Deeb, A.A. Some new retarded nonlinear integral inequalities with iterated integrals and their applications in retarded differential equations and integral equations. J. Fract. Calc. Appl. 2014, 5, 9. [CrossRef]

43. Abdeldaim, A.; El-Deeb, A.A. On generalized of certain retarded nonlinear integral inequalities and its applications in retarded integro-differential equations. Appl. Math. Comput. 2015, 256, 375-380. [CrossRef]

44. Abdeldaim, A.; El-Deeb, A.A. On some generalizations of certain retarded nonlinear integral inequalities with iterated integrals and an application in retarded differential equation. J. Egypt. Math. Soc. 2015, 23, 470-475. [CrossRef]

45. Abdeldaim, A.; El-Deeb, A.A. On some new nonlinear retarded integral inequalities with iterated integrals and their applications in integro-differential equations. Br. J. Math. Comput. Sci. 2015, 5, 479-491. [CrossRef]

46. Agarwal, R.P.; Lakshmikantham, V. Uniqueness and Nonuniqueness Criteria for Ordinary Differential Equations; Series in Real Analysis; World Scientific Publishing: Singapore, 1993; Volume 6.

47. El-Deeb, A.A. On Integral Inequalities and Their Applications; LAP Lambert Academic Publishing: Saarbrücken, 2017.

48. El-Deeb, A.A. A Variety of Nonlinear Retarded Integral Inequalities of Gronwall Type and Their Applications. In Advances in Mathematical Inequalities and Applications; Springer: Berlin, Germany, 2018; pp. 143-164. 
49. El-Deeb, A.A.; Ahmed, R.G. On some explicit bounds on certain retarded nonlinear integral inequalities with applications. Adv. Inequal. Appl. 2016, 2016, 15.

50. El-Deeb, A.A.; Ahmed, R.G. On some generalizations of certain nonlinear retarded integral inequalities for Volterra-Fredholm integral equations and their applications in delay differential equations. J. Egypt. Math. Soc. 2017, 25, 279-285. [CrossRef]

51. El-Owaidy, H.; Abdeldaim, A.; El-Deeb, A.A. On some new retarded nonlinear integral inequalities and their applications. Math. Sci. Lett. 2014, 3, 157. [CrossRef]

52. El-Owaidy, H.M.; Ragab, A.A.; Eldeeb, A.A.; Abuelela, W.M.K. On some new nonlinear integral inequalities of Gronwall-Bellman type. Kyungpook Math. J. 2014, 54, 555-575. [CrossRef]

53. Li, J.D. Opial-type integral inequalities involving several higher order derivatives. J. Math. Anal. Appl. 1992, 167, 98-110.

54. Bohner, M.; Peterson, A. Dynamic Equations on Time Scales: An Introduction with Applications; Birkhauser: Boston, MA, USA, 2001.

55. Bibi, R.; Bohner, M.; Pecarić, J.; Varosanec, S. Minkowski and Beckenbach-Dresher inequalities and functionals on time scales. J. Math. Inequal. 2013, 7, 299-312. [CrossRef]

(C) 2020 by the authors. Licensee MDPI, Basel, Switzerland. This article is an open access article distributed under the terms and conditions of the Creative Commons Attribution (CC BY) license (http:/ / creativecommons.org/licenses/by/4.0/). 